\title{
PFEM-based modeling of industrial granular flows
}

\author{
J. Cante • C. Dávalos · J. A. Hernández • J. Oliver • \\ P. Jonsén - G. Gustafsson - H.-Å. Häggblad
}

Received: 22 January 2014 / Revised: 28 February 2014 / Accepted: 5 March 2014 / Published online: 8 April 2014

(C) Springer International Publishing Switzerland 2014

\begin{abstract}
The potential of numerical methods for the solution and optimization of industrial granular flows problems is widely accepted by the industries of this field, the challenge being to promote effectively their industrial practice. In this paper, we attempt to make an exploratory step in this regard by using a numerical model based on continuous mechanics and on the so-called Particle Finite Element Method (PFEM). This goal is achieved by focusing two specific industrial applications in mining industry and pellet manufacturing: silo discharge and calculation of power draw in tumbling mills. Both examples are representative of variations on the granular material mechanical response- varying from a stagnant configuration to a flow condition. The silo discharge is validated using the experimental data, collected on a fullscale flat bottomed cylindrical silo. The simulation is conducted with the aim of characterizing and understanding the correlation between flow patterns and pressures for concentric discharges. In the second example, the potential of PFEM as a numerical tool to track the positions of the particles inside
\end{abstract}

\section{J. Cante $\cdot$ J. A. Hernández}

E.T.S. d'Enginyeries Industrial i Aeronáutica de Terrassa,

Technical University of Catalonia (UPC), Campus Terrassa UPC,

Mòdul TR5, c/Colom 11, 08222 Terrassa, Spain

J. Oliver ( $\square)$

E.T.S. d'Enginyers de Camins, Canals i Ports de Barcelona,

Technical University of Catalonia (UPC), Campus Nord UPC,

Mòdul C1, C/. Jordi Girona 1-3, 08034 Barcelona, Spain

e-mail: xavier.oliver@upc.edu

J. Cante · C. Dávalos · J. A. Hernández · J. Oliver .

International Center for Numerical Methods in Engineering

(CIMNE), C/. Gran Capitan s/n, 08034 Barcelona, Spain

P. Jonsén · G. Gustafsson · H.-Å. Häggblad

Division Mechanics of Solid Materials, Luleå University of Technology,

Luleå 97187, Sweden the drum is analyzed. Pressures and wall pressures distribution are also studied. The power draw is also computed and validated against experiments in which the power is plotted in terms of the rotational speed of the drum.

Keywords Granular flow · PFEM - Numerical modeling · Silo discharge $\cdot$ Milling

\section{Motivation}

Many industrial processes involve the manipulation and transformation of granular materials. The presence of granular flows during these processes is a strong source of problems whose origins are only partially understood. For this reason, it is important to explore and analyze via experimental and numerical models the physical mechanisms that take place when granular flows are present. This work focuses on two industrial problems related to pellet manufacturing in mining industry: the silo discharge and the tumbling mills.

\section{Silo discharge modeling}

Granular material behavior in silos has been a topic of interest since late nineteenth century; this is due to the wide number of problems that are present during its storage and discharge. The problems found are not only on the structure itself but also on the material. The problems involve instabilities on the foundations, buckling of silo walls, blockage of the material at the outlet, and discharge overpressures [1].

Internal pressures and the pressures exerted on the silo walls are strongly influenced by the flow patterns exhibited by the material during its discharge [2]. These flow patterns can be broadly classified as either mass flow or funnel flow [3]. Mass flow patterns are smooth and relatively uniform, with velocity profiles exhibiting highest values at the center- 
line of the outlet, and decreasing progressively toward the silo walls. Mass flow patterns presents a depression on the bulk of the material since the discharge is faster at the center than at the outer portions. On the other hand, funnel flows are characterized by a stagnant zone toward the silo walls, a region of vertical motion in the center of the outlet, and a transition or jamming zone [2]. The definition of mass flow or funnel flow is a function of the material properties, the geometry of the silo, and the interaction between the silo's wall and the granular material.

Experimental tests show that for large height to breadth ratios the mass flow is more predominant, while for lower ratios the funnel flow is more frequent [1]. It is also observed that funnel flow tends to form for large hopper angles and large wall friction coefficients, while for smaller values the predominant flow is a mass type.

The pressures during discharge are strongly influenced by the flow regime. It is important to understand and define the pressures since they have repercussions on the structure of the silo and its functionality [4]. Therefore, it is essential to establish models to predict these conditions. Unfortunately, this is not a trivial task, neither on experimental nor numerical models. Experimental models have the drawback of the affectation on the response due to the scale factor, and the difficulties due to the instrumentation required, for example in the case of obtaining the flow patterns, since walls are opaque and there is no devise able to directly measure and observe those patterns.

\section{Tumbling mills modeling}

The second industrial application herein presented is the milling of granular material in a tumbling drum. Comminution of the material consumes $50 \%$ of the total mineral processing cost [5]. Studies have found that grinding of granular material in tumbling mills are inefficient, since large amount of energy is wasted in unproductive impacts-in the sense that such impacts do not break particles [6]. The study of charge motion during the milling process allows us to understand how energy is consumed and which efforts could be done in order to optimize the operating conditions of the drum.

As may be surmised, this optimization task is a rather difficult one, mainly because material behavior within the drum is poorly understood. Different techniques have been proposed in order to monitor the performance of a tumbling mill. Among them, the use of mill noise and mill vibrations, to measure the degree of filling [7], the force exerted by the material on the lifters [6], and the power readings during the grinding process, to interpret the filling degree of the material [5], are found. Measuring the driving torque and relate it to the process by numerical models can be one possible way to validate, control, and optimize the grinding system. Since the numerical model herein presented is developed in the framework of continuum mechanics, it is straightforward the calculation of the system energy, via the balance equations, for its validation with experimental results.

It is important to have in mind the complex nature of the milling process when creating models. To decrease the gap between model and reality, more physically precise models are necessary. Measurements are important for improving the milling efficiency and gaining more understanding of the process itself. A step towards a more physically correct numerical description of mill systems was the combined DEM-FEM model presented by [8]. With the DEM-FEM model forces and mechanical waves as well as structural responses and their influence on the charge motion can be studied. The model gives the opportunity to optimize the material selection of the mill structure. In the work by [9] a combined SPH-FEM model was used in simulations of tumbling mill processes.

Therefore, it is reasonable to expect that numerical modeling of both problems - the silo discharge and the tumbling mill-can offer the possibility to explore the effect of input parameters that are not easily accessible to experimentation.

Several numerical strategies have been proposed over the last few years to study granular flows. Hard spheres seem the simplest way to simplify the level of difficulty in modeling granular material flow. In this case, collisions are so brief that penetrations are not considered and the loss of momentum is characterized by means of a coefficient of elastic restitution. This approximation is the basis of the so called 'collisional' or 'event-driven' (ED) models [10]. In contrast, the soft sphere approximation is based on the penetration depth. The interaction force between spheres depends on this magnitude.

Representative models in this group are based either on the molecular dynamics (MD) or on the distinct element (DEM) methods. Both methods are identical in spirit: penetration is detected when the distance between the centers of two spheres is less than the sum of their radii. In this case, a penetration force, defined in terms of contact and friction models, is activated. Coulomb law is usually used as a friction model though more complex interaction can also be included. The motion of the spheres is governed by means of the Newton's second law and the method of time integration is explicit. The consequence, is that the time step must be below a critical value, otherwise the solution can diverge due to numerical instability [11]. Recent versions of DEM extend the interaction to complex polyhedral blocks.

The different methods described above have in common the discrete character of the approximation: a sphere represents a small quantity of grains and its motion is traced by classical dynamics.

The other point of view-the one adopted in the present paper-is the continuum approximation. In this case, the granular material is assumed to behave as a continuum that can be described by means of a constitutive model and different continuous field variables. At this point, the key question 
is how to obtain the constitutive model and, for many years, a large number of researchers have tried to answer this question. Among the different proposals, two families of models can be clearly distinguished, i.e. micromechanics and phenomenological models. In the first family, the stress field is the result of a study that comes from molecular dynamics. The second one involves all the models based on continuum mechanics, which in turn can be divided in terms of the formulation, i.e. Lagrangian or Eulerian. In the Lagrangian formulation (or description), the variables are expressed in terms of the initial configuration. The consequence, in terms of a finite element mesh, is that the element distortion is so high that accuracy decreases drastically and even the computation can be interrupted abruptly. These problems explain why a large family of continuum models, for granular materials, is formulated in Eulerian description. In this case, the granular material is treated as a non-Newtonian viscous fluid, in which the balance momentum equations are verified over a fixed undistorted finite element mesh. Serious limitations are found when the method is needed to handle complicated boundary conditions, as is the case of the free surface presented during the flow of granular materials.

To circumvent these problems, a Lagrangian based model, in the frame of PFEM [12], is proposed herein. The spatial discretization of the corresponding variational equations is formulated in the new framework, in which the nodes of the spatial domain are continuously reconnected by the action of a Delaunay triangulation. In contrast to classical PFEM approximations, in which the free boundary is obtained by a geometrical technique (alpha shape method), in this work the boundary is treated as a material surface, where the boundary nodes are removed or inserted by means of an error function.

The constitutive model is based on the concepts of plasticity at large strains. The yield surface is defined in terms of a Drucker Prager yield function characterized, in turn, by two constitutive parameters (the cohesion and the internal friction coefficient), and provided with a deviatoric plastic flow. The elastic part is defined by a hypoelastic model. These expressions are accompanied by the corresponding governing equations and boundary conditions.

The remaining of the paper is organized as follows. Section 2 is devoted to the description of the numerical model, we start with a brief description of PFEM and the modifications introduced here concerning insertion and remotion of particles. The section continues with a description of the proposed constitutive model, elastic response, yield criteria, and flow rule, among others. Section 3 focuses on two industrial applications: silo discharge and power draw prediction in tumbling mills. Both examples are representative of variations on the granular material mechanical response-varying from a stagnant configuration to a flow condition. Finally, in Sect. 4, some concluding remarks are presented.

\section{Numerical model}

\subsection{The Particle Finite Element Method}

The Particle Finite Element Method (PFEM) is a FEM-based particle method [12], initially proposed for the solution of the continuous fluid mechanics equations. The main objectives were, on the one hand, to develop a method to eliminate the convective terms in the governing equations, and, on the other hand, the introduction of a technology, based on the alpha shape method used in other areas, able to deal with free boundary surfaces. The interpretation of the method has evolved from a meshless method, in which the nodes are supposed to be particles that move according to simple rules of motion, to a sort of updated lagrangian approach in which the advantages of the standard FEM formulation for the solution of the incremental problem are used.

In the PFEM approach, the continuum material is modelled using an updated lagrangian formulation, and the finite element method is used to solve the variational incremental problem. Hence, a mesh, discretizing the domain, is generated in order to solve the governing equations. In this context, discretization nodes can be regarded as material points whose motion is followed during the time stepping solution. A typical solution with the PFEM contains the following steps.

1. Discretize the domain with a finite element mesh. The mesh generation is typically based on a standard Delaunay triangulation.

2. Identify the external boundaries of the domain. In this step the Alpha Shape method [13] is used for the boundary recognition.

3. Solve the variational incremental problem. The solution provides the state variables at each time step: displacements, velocities, pressure, and stresses, among others.

4. Update the nodal position with the displacement values obtained in step 3 .

5. Generate a new mesh. The mesh results from the Delaunay triangulation of the updated position of the nodes.

6. Go back to step 2 and repeat the solution process for the next time step.

The continuous reconnection introduced in step 5 is the key strategy to circumvent the typical mesh distortion generated when a Lagrangian description is used with problems involving large strains.

Although PFEM was initially applied to problems in the field of fluid mechanics, it is being currently applied to a wide range of simulation problems [14-17]: filling, erosion, mixing processes, thermo-viscous processes and thermal diffusion problems, among others. First applications of PFEM to solid mechanics are found in [18] to problems involving large strains and rotations, multi-body contacts and creation of new surfaces (riveting, powder filling and machining). 
Applications to the response of rockfill dams on overtopping conditions, via a non-Newtonian fluid, can be found in [19]. Recent applications to geomechanical problems can be found in [3]. In this work, a rigid-plastic model was adopted and the governing equations were formulated in terms of a variational principle to facilitate a straightforward solution via mathematical programming techniques.

In the present work, we extend the application to the numerical simulation of granular flows via a viscoelastoplastic model.

The standard PFEM presents some weaknesses when applied to granular flow simulation. The boundaries generated using alpha-shape method may adversely affect the predictive capacity of the model in many situations and aspects: lack of mass conservation; absence of equilibrium on the boundary due to the introduction of artificial perturbations and generation of unphysical welding of the granular material and the walls of the container. To deal with these problems, we propose in this work a free-surface construction method that uses a constrained Delaunay triangulation combined with procedures aimed at improving the quality of the new mesh (by insertion, remotion and reposition of the nodes). These procedures are applied locally and not in every time step. Specific size metrics control node insertion and remotion, while Laplacian smoothing algorithm drives the repositioning of nodes.

\subsection{Continuum modelling of dense granular flows}

The assumption of modelling a discrete media as a continuum has been subject of extensive study. It has been demonstrated that the differential equations used to define the kinematics of their deformation are correct as the number of particles increases $[10,20]$. The continuum approach allows us to interpret the particle interaction as a transmission of energy through the domain by a mathematical formulation. Under some considerations, the microscopic relations of the particles are properly transformed into macroscopic equivalents in terms of the material properties.

The definition of a unique model capable of representing any physical condition for any material is difficult to establish (if not impossible). Granular materials models are not an exception in this regard. In what follows, we present a constitutive model for dense granular flows able to differentiate stagnant and flow zones as well as the jamming transition between these conditions.

The model is formulated in the framework of large deformation plasticity theory. The yield function is defined in stress space by a Drucker-Prager yield surface equipped with a deviatoric flow rule. This plastic flow condition is considered nearly incompressible so the proposal is integrated in a $u-p$ mixed formulation with the pressure stabilization proposed in [21].

\section{Model limitations}

It is important to narrow the flow conditions and granular materials that the model is intended to represent. The model is proposed for dense granular materials, aimed at characterizing the static condition of the materials at rest, as well as for slow granular flows including its jamming transition. The statement of dense granular flows implies small velocities in the material as well as the assumption for the granular particles to remain in contact while the material spreads or flows. The mechanical properties of dense granular materials are governed by the frictional interaction between the particles, which means that the deformations that the material experiences while flowing do not present significant volumetric variations; this fact allows one to assume that the material is nearly incompressible. In this sense, the model is not suitable for industrial processes where granular materials are subject to compaction or to dispersion.

In order to represent a frictional response between particles, the presence of any other material between the grains is ignored. The isotropy assumption of the model is conserved maintaining a high homogeneity in size and shape of the granular particles. Even so, this assumption is achieved when the particle size is considerable small compared to the size of the domain under consideration.

\subsection{The constitutive model}

\subsubsection{Kinematics of plastic large deformations}

Consider the granular material as a deformable body $B$ consisting of continuously distributed material occupying, at a reference time $t_{0}$, a region $\Omega_{0}$ of $\mathbb{R}^{n_{d}}\left(n_{d}=2,3\right)$. The deformation at time $t$ relative to the reference configuration is given by a smooth mapping $\varphi_{t}: \Omega_{0} \rightarrow \Omega_{t} \subset \mathbb{R}^{n_{d}}$. Let $\mathbf{V}=\partial \varphi_{t} / \partial t$ and $\mathbf{A}=\partial \mathbf{V} / \partial t$ be the material velocity and the material acceleration, respectively; and let denote by $\mathbf{v}(\mathbf{x}, t)$ and $\mathbf{a}(\mathbf{x}, t)$ the spatial counterparts of $\mathbf{V}$ and $\mathbf{A}$, respectively. On the other hand, let $\nabla \mathbf{v}$ bet he spatial velocity gradient and $\mathbf{d}=\frac{1}{2}\left(\nabla \mathbf{v}+\nabla \mathbf{v}^{T}\right)$ the symmetric part of $\mathbf{d}$, termed the spatial rate of deformation tensor.

As is customary in the literature of large plasticity theory, we adopt the assumption of the local multiplicative decomposition of the deformation gradient of $\boldsymbol{\varphi}_{t}$, denoted by $\mathbf{F}$, into plastic and elastic parts:

$\mathbf{F}=\mathbf{F}^{e} \mathbf{F}^{p}$

where $\mathbf{F}^{p}$ represents a pure plastic deformation from $\Omega_{0}$ to a certain stress-free intermediate configuration [22], and $\mathbf{F}^{e}$ a pure elastic loading from such an intermediate configuration to the current configuration $\Omega_{t}$. Likewise, we shall denote by $J=\operatorname{det} \mathbf{F}$ the determinant of the deformation gradient. 
For the particular case of granular material flows, which is the one that concerns us, it is also assumed that elastic deformations are, comparatively with the total ones, small. We shall thus limit our considerations to a kinematic description that considers arbitrarily large plastic deformations and small elastic strains. Under such circumstances, the distinction between the abovementioned intermediate state and the current configuration $\Omega_{t}$ becomes negligible, and tensorial fields defined naturally at the intermediate configuration can be transformed directly into spatial tensorial fields. A valuable implication of this is that the Almansi strain tensor $\mathbf{e}(\mathbf{x})=1 / 2\left(\mathbf{1}-\mathbf{F}^{-T} \mathbf{F}^{-1}\right)$ inherits the additive structure of classical small strain formulations [23]:

$\mathbf{e}=\mathbf{e}^{e}+\mathbf{e}^{p}$

being $\mathbf{e}^{p}=1 / 2\left(\mathbf{1}-\mathbf{F}^{p^{-T}} \mathbf{F}^{p^{-1}}\right)$ and $\mathbf{e}^{e}$ the plastic and elastic parts, respectively, of the Almansi strain tensor. This property that, incidentally, holds also for the rate of deformation tensor:

$\mathbf{d}=\mathbf{d}^{e}+\mathbf{d}^{p}$

where $\mathbf{d}^{e}$ and $\mathbf{d}^{p}$ are the elastic and plastic parts of rate of deformation tensor, respectively, will afford a remarkable aspect of simplicity in deriving the ensuing constitutive equations.

\subsubsection{Elastic response}

In many industrial applications, involving granular flows, the size and localization of stagnant zones formation determines the flow behavior. The correct determination of these zones requires the introduction of a specific model tool. We propose the formulation of an elasto-plastic model capable of predicting stagnant zones and flow regions simultaneously in any spatial domain. For the elastic response, we propose a class of model of phenomenological, rate independent, plasticity obtained by an ad hoc extension of the infinitesimal theory, which relies on a hypoelastic characterization of the elastic behavior. The advantage of this model lies on the conceptual simplicity of its formulation. We write the elastic response in the following form:

$L_{v} \boldsymbol{\tau}=\mathbf{c}:\left[\mathbf{d}-\mathbf{d}^{p}\right]$

where $L_{v}(\cdot)$ denotes the Lie objective stress rate, and $\tau$ denotes the Kirchhoff stress tensor. We assume that the spatial elasticity tensor $\mathbf{c}$ is given by:

$\mathbf{c}=2 \mu\left(\mathbf{I}-\frac{1}{3} \mathbf{1} \otimes \mathbf{1}\right)+\kappa \mathbf{1} \otimes \mathbf{1}$

where I and $\mathbf{1}$, with components $I_{a b c d}=\left[\delta_{a c} \delta_{b d}+\delta_{a d} \delta_{b c}\right] / 2$ and $1_{a b}=\delta_{a b}$, are the fourth and second order symmetric unit tensors, respectively. The parameters $\mu$ and $\kappa$ represent the shear and bulk elastic moduli, respectively. In the case of general granular materials, these parameters are not constant but depend on a hardening internal variable [24,25]. For iron pellets, and according to experiments conducted by Gustafsson [26], the Poisson's coefficient can be regarded, in a first approximation, as constant $(v=0.21)$; by contrast, $\kappa$ is strongly influenced by the level of isostatic compression. The following law corresponds to the fitting equation to iron pellet bulk's modulus values, obtained in instrumented confined compression tests carried out by Gustafsson [26,27]:

$\kappa(p)=a_{1}+a_{2} p$

where $p$ represents the isostatic pressure, with $a_{1}=$ $60.0 \mathrm{MPa}$ and $a_{2}=150.0$.

\subsubsection{Yield condition}

The yield condition used in this work is derived, essentially, from the Drucker-Prager type criterion. This yield condition is formulated in terms of the Kirchhoff stress invariants $p=$ $\frac{1}{3} I_{1}=\frac{1}{3} \operatorname{tr} \tau$ (mean stress), and $q=\sqrt{2 J_{2}}=\sqrt{\operatorname{dev} \tau: \operatorname{dev} \tau}$ (norm of deviatoric stress); its envelope is determined by the following equation:

$\phi(\tau)=q+b_{1} p-b_{2}=0$

This equation represents a straight line in the $p-q$ plane. Parameter $b_{2}$ is customarily referred to as cohesion, and it can be interpreted as the shear strength under zero hydrostatic stress (intersection of the Drucker-Prager yield surface with the $q$-axis); $b_{1}$ represents the slope of the Drucker-Prager line, and it is termed the parameter of internal friction. With experimental values of cohesion and the frictional coefficient, one can plot $q$ as a function of $p$ when the material yields. To achieve this, different cylindrical triaxial tests can be carried out: (1) conventional triaxial compression and conventional triaxial extension, with the stress path having a positive slope, (2) triaxial compression, triaxial extension, and simple shear, with vertical stress paths and, finally, (3) reduced triaxial compression, and reduced triaxial extension, with a stress path with a negative slope. Gustafsson et al. [26] performed various sets of compression and shear tests on iron ore pellets, and arrived at $b_{1}=1.0$ and $b_{2}=0$ as numerical values for those parameters.

\subsubsection{Flow rule}

As is customary in the framework of incremental plasticity theory, we apply the concept of flow rule to obtain the plastic rate of deformation tensor $\mathbf{d}^{p}$ in terms of the plastic flow vector $\mathbf{m}$ associated to the yield surface:

$\mathbf{d}^{p}=\dot{\lambda} \mathbf{m}$

The positive scalar factor $\dot{\lambda}$, referred to as consistency parameter or plastic multiplier must obey the standard Karush- 
Kuhn-Tucker loading/unloading conditions, that in the context of rate-independent plasticity read:

$\dot{\lambda} \geq 0, \phi(\tau) \leq 0, \quad$ and $\quad \dot{\lambda} \phi(\tau)=0$

along with the consistency condition $\dot{\lambda} \dot{\phi}(\tau)=0$ [22]. Furthermore, each plastic flow vector $\mathbf{m}$ is presumed to be aligned with the gradient of a certain plastic potential function, $\varphi$, i.e. $\mathbf{m}=\partial \varphi / \partial \tau$. In this work, the following plastic potential is proposed:

$\varphi(\tau)=\frac{1}{2}\|\operatorname{dev} \tau\|^{2}$

Therefore, the plastic rate of deformation tensor can be written as

$\mathbf{d}^{p}=\dot{\lambda} \operatorname{dev} \tau$.

Since $\mathbf{d}^{p}$ is proportional to a deviatoric tensor, the flow rule asserts plastic incompressibility. While, as noted in [28], dilation in dense flow does occur, it is typically on the order of only a few percent and quickly reaches a steady value over large deformations. Moreover, relative density (or packing fraction) is not necessary for purposes of computing flow motion; hence, the approximation of plastic incompressibility should have negligible effect on the velocity field of a dense granular flow. Note that this approximation affects only the stress state in the static regions-hydrostatic stresses are fully elastics.

Equations (4), (7), (9), and (11) define the elasto-plastic part of the proposed model for granular flows.

\subsection{Visco-plastic regularization}

As pointed out in the introduction, constitutive equations for granular material flows are still a matter of debate. One difficulty is that granular material can behave like a solid (in a powder heap), a liquid (when poured on an inclined plane) or a gas (when strongly stirred up). For the solid-like state, the elasto-plastic constitutive model described in the foregoing is proposed. However, the intermediate flow regime, where the granular material flows like a liquid, as in a silo discharge, requires the inclusion of viscous behavior. The fundamental features of granular flows in this regime are: a yield condition (a shear stress limit below which the grain doesn't move) and general nonlinear dependence on shear rate when flowing. In this sense, granular behavior shares similarities with classical visco-plastic models. Here we propose a visco-plastic regularization via a Duvaut-Lions type model. The new flow rule reduces to

$\mathbf{d}^{v p}=\frac{1}{\tau_{R}} \mathbf{c}^{-1}:\left[\tau-\tau^{e p}\right]$

where $\tau_{R}$ refers to the relaxation time and $\tau^{e p}$ corresponds to the solution of the elasto-plastic part of the model (described
Box 1 Visco-elasto-plastic model for dense granular material

Elastic response

$$
L_{v} \boldsymbol{\tau}=\mathbf{c}:\left[\mathbf{d}-\mathbf{d}^{v p}\right]
$$

Yield function and elastic domain in stress space

$$
\begin{aligned}
& \phi(\boldsymbol{\tau})=\|\operatorname{dev} \boldsymbol{\tau}\|+b_{1}\left(\frac{1}{3} \operatorname{tr} \boldsymbol{\tau}\right)-b_{2} \\
& E_{\hat{\tau}}=\{\hat{\boldsymbol{\tau}} / \phi(\boldsymbol{\tau}) \leq 0\}
\end{aligned}
$$

Flow rule

$$
\mathbf{d}^{v p}=\frac{1}{\tau_{R}} \mathbf{c}^{-1}:\left[\boldsymbol{\tau}-\boldsymbol{\tau}^{e p}\right]
$$

$\boldsymbol{\tau}^{e p}$ is solution of the following sub-problem

$$
\begin{aligned}
& L_{\nu} \boldsymbol{\tau}^{e p}=\mathbf{c}:\left[\mathbf{d}-\mathbf{d}^{p}\right] \\
& \mathbf{d}^{p}=\dot{\lambda} \operatorname{dev} \boldsymbol{\tau}^{e p} \\
& \dot{\lambda} \geq 0, \quad \phi\left(\boldsymbol{\tau}^{e p}\right) \leq 0, \quad \dot{\lambda} \phi=0
\end{aligned}
$$

in the foregoing). Box 1 summarizes the constitutive model proposed in this work to describe the behavior of a dense granular material.

\subsection{Governing equations}

Next, we summarize the equations of motion, in local form, relevant to numerical implementation in the framework of PFEM. The local equations of motion in the Eulerian description take the form:

$\operatorname{div} \boldsymbol{\sigma}+\rho \mathbf{b}=\rho \mathbf{a}$ in $\Omega_{t}=\varphi_{t}\left(\Omega_{0}\right)$

where $\boldsymbol{\sigma}=\frac{1}{J} \tau$ is the Cauchy stress tensor, $\rho$ is the density in the current placement, $\mathbf{b}$ is the body force per unit of volume in $\Omega_{t}$ and div (.) denotes the divergence of $\sigma$. We let $\partial \Omega_{t}$ be the boundary of $\Omega_{t}$ and assume that the deformation is prescribed on $\partial_{\varphi} \Omega_{t} \subset \partial \Omega_{t}$ as

$\varphi_{t}=\bar{\varphi}_{t} \quad$ (prescribed) on $\partial_{\varphi} \Omega_{t}$

whereas the nominal traction vector $\mathbf{s}$ is prescribed on $\partial_{s} \Omega_{t} \subset$ $\partial \Omega_{t}$ as

$\mathbf{s}=\overline{\mathbf{s}} \quad$ (prescribed) $\quad$ on $\partial_{s} \Omega_{t}$

Now, we summarize the weak formulation of momentum balance equations as a first stage toward the numerical implementation within the framework of PFEM.

We let $V_{\varphi}$ be the space of admissible displacements

$V_{\varphi}=\left\{\mathbf{v}: \varphi_{t} \rightarrow \mathbb{R}^{n_{d}} / \mathbf{v}(\mathbf{x})=0\right.$, for $\left.\mathbf{x} \in \partial_{\varphi} \Omega_{t}\right\}$

we call $\mathbf{v} \in V_{\varphi}$ an admissible displacement. Taking the dot product with any $\mathbf{v} \in V_{\varphi}$, integrating over $\Omega_{t}$, and using the divergence theorem, we get 


$$
\begin{array}{r}
-\int_{\varphi_{t}(\Omega)} \sigma: \nabla \mathbf{v} d \Omega+\int_{\varphi_{t}(\Omega)} \rho \mathbf{b} \cdot \mathbf{v} d \Omega \\
\quad+\int_{\partial_{\varphi} \Omega_{t}} \bar{s} \cdot \mathbf{v} d s=\int_{\varphi_{t}(\Omega)} \rho \mathbf{a} \cdot \mathbf{v} d \Omega
\end{array}
$$

that is the integral form of the momentum balance equation in its spatial form. The constitutive model described in Box 1, the system of Eqs. (17), the boundary condition (14), and the corresponding initial conditions, define the whole continuum problem to be solved. Unfortunately, due to the inherent incompressible character of the proposed deviatoric flow rule, the so-called locking phenomena may emerge when solving the weak form of the motion equation using standard, irreducible formulations. In order to circumvent this problem, a mixed formulation, stabilized using the polynomial pressure projection [21], is proposed. In this case, the Cauchy stress tensor in Eq. (17) is decoupled into its deviatoric $\sigma^{d}$ and volumetric parts

$\boldsymbol{\sigma}=\boldsymbol{\sigma}^{d}+p \mathbf{1}$

where $p$ is a new unknown of the problem that represents the pressure. The weak form of the new additional equation reads

$$
\int_{\varphi_{t}(\Omega)} \delta q\left(\frac{1}{J} \tau_{m}-p\right) d \Omega=0
$$

for any $\delta q$, square-integrable function over $\varphi_{t}(\Omega) . \tau_{m}$ denotes the media stress of the Kirchhoff stress tensor $\tau$ (solution of the constitutive model summarized in Box 1). Equation (19) is completed with the addition of a stabilization term, proposed by Bochev in [21] and here extended for its use in large strains contexts. The inclusion of this last Eq. (19) defines the ultimate continuum problem to be solved.

\subsection{Numerical implementation}

Although the thorough treatment of issues pertaining to the discretization and numerical implementation of the model is not the goal of this work, it is convenient to, before launching into details of the validation of the model, provide a brief overview of some aspects that may result crucial for acquiring a proper grasp of the ensuing computed results. The algorithm used for integrating the constitutive differential equations is the so-called Impl-Ex scheme, originally proposed by Oliver et al. [29], and further elaborated in Oliver et al. [30], to improve the robustness of implicit algorithms in the numerical simulation of material failure. They coined the term Impl-Ex (IMPLicit-EXplicit) to suggest that the method shares some of the features of both implicit and explicit integration schemes. Roughly, the essence of the method is to solve explicitly for some variables (in the sense that the val- ues at the beginning of the increment are presumed known) and implicitly for other group of variables. This methodology, in turn, entails the solution at each time increment of the non-linear system of equations stemming from the implicit backward-Euler difference scheme, i.e. the so-called return-mapping equations, since the above mentioned group of "explicit" variables are but extrapolated values of the same quantities computed implicitly at previous time steps.

In regard to the finite element approximation, an updated lagrangian viewpoint has been adopted for describing the motion of the mesh, with a mesh update procedure based on the so-called Particle Finite Element Method (PFEM). This method imposes a limitation concerning element technology: finite elements are to be three-noded triangular elements (linear). To avoid the locking problem that may emerge in the response in using such simple finite elements, a finite element approximation based on a mixed variational formulation, with displacements and pressure as basic variables, and continuous linear interpolation for both fields has been implemented [21]. The formulation has been specialized to address plane strain and axisymmetric problems.

Tools are modeled as rigid bodies. Granular-tool frictioncontact conditions are imposed using the so-called Contact Domain Method described in Oliver [31], Hartmann [32] in which interacting portions of contacting bodies are identified via an interface mesh. The interface mesh, having zero thickness, has the same dimension as the contacting bodies, and provides a complete, continuous and non-overlapping, pairing of the contact surfaces.

The contact domain can be easily incorporated into the framework of PFEM, overcoming some deficiencies of the node-to-segment pairings, like dependence on the choice of master or slave faces and over-constraining of some specific parings. The displacement field on the contact domain is naturally taken from the interface of the contacting bodies and the definition of the strain measures is straightforward. The core of the method is to discretize the domain within the contacting bodies via a constrained Delaunay triangulation, to allow the accurate definition of the gap within the active contact area. The contact boundary condition is then enforced in the continuum by the use of Nitsche's technique. The resulting method was proved to be robust and efficient for a variety of demanding problems in computational mechanics, and herein is proposed to solve the considered industrial granular flow applications.

\section{Industrial applications}

This section focuses on two industrial applications silo discharge and power draw prediction in tumbling mills. Though the considered granular material is here iron pellets, both examples are representative of the mechanical response - 
Fig. 1 Full-scaled experimental silo [4,33]: a Elevation view (all dimensions in $\mathbf{m m}$ ); $\mathbf{b}$ seeding of radio tags per levels

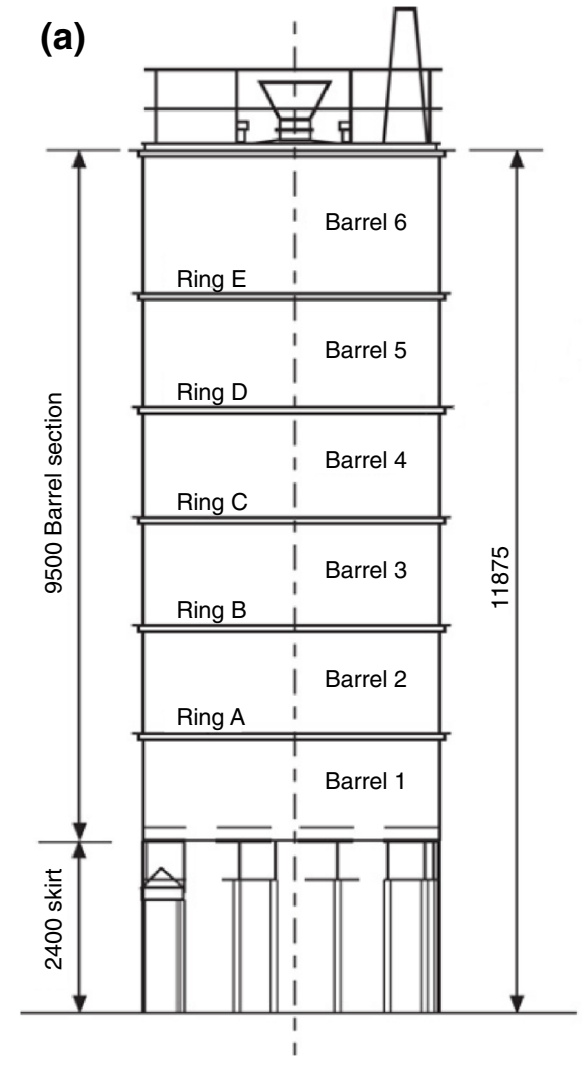

(b)

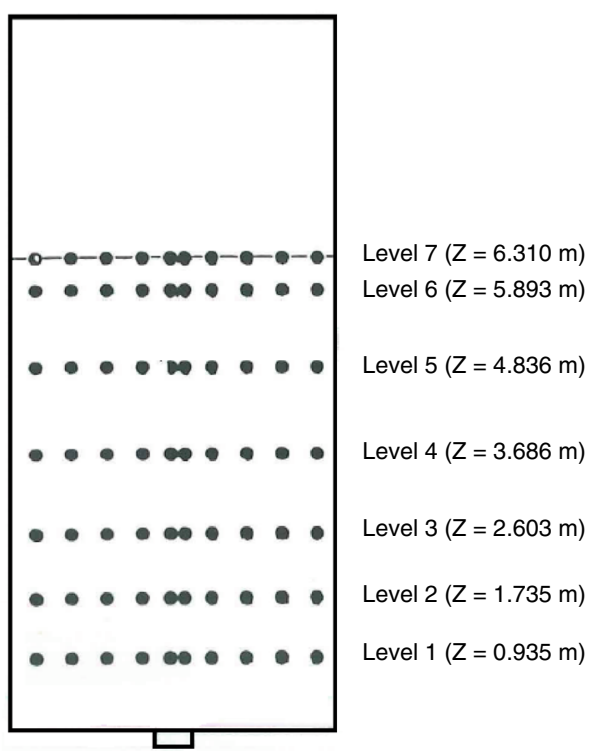

varying from a stagnant configuration to a flow conditionfor a large variety of granular materials.

The silo discharge is validated using the experimental data, collected by Rotter et al. [33], on a full-scale flat bottomed cylindrical silo. The simulation is conducted with the aim to characterize and understand the correlation between flow patterns and pressures for concentric discharges. Numerical and experimental flow patterns are compared for different levels of discharge. The effect on the flow patterns due to variation of the mechanical properties and the outlet diameter is also analyzed. In the second example, the potential of PFEM as a numerical tool to track the positions of the particles in the interior of a rotational drum is analyzed. Pressures and wall pressures distribution are also studied. The power draw is also computed and validated against experiments in which the power is plotted in terms of the rotational speed of the drum.

\subsection{Silos discharge: experimental setup}

The importance of this example lies on the use of a full-scaled silo; since it is identified the influence on the experimental response given by the scale of the model $[1,4]$. The model is a full-scaled flat-bottomed cylindrical silo, instrumented to study the flow patterns and their correlation with the wall pressures. Several conditions of discharge and materials were tested.
The silo is 4,200 $\mathrm{mm}$ in diameter with 9,500 $\mathrm{mm}$ high barrel section; see Fig. 1(a). It has three $480 \mathrm{~mm}$ diameter outlets: one concentric, one fully eccentric and one half way between. Each outlet has a hydraulically controlled slide gate, which operates from one side. This slide gate is opened partially, which makes the outlet in a shape of a circular segment.

In the experiment, radio tags were placed in the silo with the aim of predicting flow patterns during the discharge. The radio tags were carefully located along and across the material. A total of 280 radio tags were placed uniformly at seven different levels and eight spokes for each level separated at five different radii, see Fig. 1(b). The silo was filled concentrically to avoid localization of pressures due to particle packing. The filling of the material was stopped at each level and the bulk was raked in order to dispense a flat surface; afterward, the template with the seeding position was located to define the exact position of the tags. In order to avoid damage of the radio tags, they were placed inside tennis balls. The logging system for the tags consisted on an aerial wired straight into an amplification box. The signal was processed in order to identify the radio tag at the time that it was expelled through the outlet.

The flow patterns were calculated via an extrapolation of the position of the tags and their time residence. For the position of the tags along the discharge, the authors (of the experiment) define the assumption of a trajectory defined by the shortest path, which for many markers and mass 
flow it is correct; and for the velocity it was determined an exponential changing velocity supported by the time residence of the markers. It was observed that even for concentric discharge, a full symmetric pattern was not obtained; however, the difference is too slight to be considered significant.

The experiment results are taken from the case corresponding to a concentric discharge of iron ore pelletsseveral technical aspects of the experiment, described as PCB test, are reported in Ref. [33]. The silo was filled concentrically and the solid surface raked flat at a mean height of $6,400 \mathrm{~mm}$.

The mechanical characterization of this material was carefully analyzed by Gustafsson, in Ref. [26]. The most relevant mechanical properties are: the particle size has a range between $12-15 \mathrm{~mm}$, the bulk density is $2.3 \mathrm{~kg} / \mathrm{m}^{3}$, the reported internal friction coefficient is 0.67 , the bulk modulus is $32 \mathrm{Mpa}$, and the wall friction between iron ore pellets and steel plate is 0.6 .

\subsection{Silos discharge, numerical simulation}

The computational model is a flat-bottomed cylindrical silo with a concentric outlet. The dimensions and filling height are based upon the full-scale experiments by Rotter. The diameter of the silo is $4,200 \mathrm{~mm}$ and the filling height 6,400 $\mathrm{mm}$. A full opening outlet of $480 \mathrm{~mm}$ diameter is used as a reference example. The simulation is performed with the PFEM method described above using an axisymmetric formulation. The edge of the outlet is rounded-using a radius of $40 \mathrm{~mm}$-in order to reduce geometrical singularities. A non-uniform discretization of 6,250 nodes is used to describe the initial configuration of the pellets domain; each node represents 3 degrees of freedom ( 2 for displacements and 1 for the pressure) as consequence of using a mixed formulation. The non-uniform discretization is fundamental for the simulation, since the opening of the outlet is considerable small compared to the size of the silo. The average element size is $100 \mathrm{~mm}$, except near the outlet, where the element size is $25 \mathrm{~mm}$, approximately.

In all the examples, calculations have been made with the following material data: bulk density $\rho=2,300 \mathrm{~kg} / \mathrm{m}^{3}$, bulk modulus $\kappa=60 \mathrm{MPa}$, shear modulus $G=7 \mathrm{MPa}$, internal friction coefficient $b_{1}=1$ (where the internal friction coefficient is $30.57^{\circ}$ ), cohesion $b_{2}=0$, relaxation time $\tau_{R}=2 \times 10^{-7} \mathrm{~s}$, Coulomb's dry friction $\mu=0.6$. The simulations has been run with a time-step length $\Delta t=2 \times 10^{-4} \mathrm{~s}$. The total time analyzed is $95 \mathrm{sec}$, corresponding to a volume silo discharge of $20 \mathrm{~m}^{3}$. To get a steady start after the gravity is applied the outlet is closed the first $0.5 \mathrm{~s}$ of the simulation time.

The PFEM reconnecting process is one of the basic tasks that allow the method to handle large deformation processes. Nevertheless, it was necessary to introduce additional criteria in the remeshing and reconnecting process to define a spatial discretization with a sufficiently high degree of quality. These criteria allow the finite element mesh having a more homogeneous distribution of the nodes and constraining the boundary in order to define a material surface.

The domain discretization into particles is a continuous process, repeated for these simulations every five time steps. The remeshing process covers three main steps: insertion (and remotion) of particles, particles reposition, and mesh information transference; this process is carried out after solving the system equations and updating the coordinates of the mesh.

The insertion of particles is carried out in those elements attaining a size higher than a reference tolerance; the particle is inserted at the center of the element in inner elements. In boundary elements, on the other hand, new nodes are introduced if the distance between two adjacent nodes exceeds the prescribed tolerance. The elimination of nodes takes into account the average size of those elements adjacent at the concerned node; if the averaged size is smaller than a reference tolerance, the node is removed. In the case of boundary nodes, if the distance between two adjacent nodes is smaller than the tolerance both nodes are removed and a new one is placed in the middle.

Repositioning of particles is a local procedure taking into account the mesh quality of the patch of elements connected to a single particle and it is made in terms of the size and shape metrics defined in Ref. [34]. For those nodes connecting elements with a higher degree of distortion, particles are repositioned using a standard Laplacian smoothing. Finally, mesh information transference is carried out by mesh projection; it should be noted that this method is different from that employed in classical PFEM (a smoothing process defining nodal variables).

The initial, intermediate and final configurations of the numerical silo are shown in Fig. 2.

\subsubsection{Flow patterns comparison}

To compare the numerical results with the experiment, seven horizontal lines in the numerical silo are marked and traced through the solution. These horizontal lines coincide with the levels where the radio tags were placed, see Fig. 1(b). The positions at three different volumes of discharge are compared with the computer visualizations of the experiment in Fig. 3. The blue lines stand for the numerical results and the red cross marks for the computer visualization of the tags position. The comparison is extended to $17 \mathrm{~m}^{3}$ of material discharged.

In general, the trends between numerical results and the computer visualization of the tags are similar in most of the 
(a)

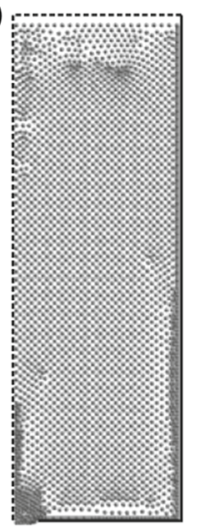

(b)

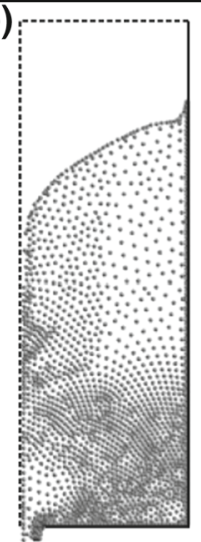

(c)

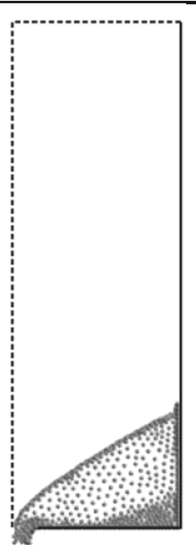

Fig. 2 Particle discretization of the domain at different stages of the silo discharge: a Initial outlet opening, b $20 \mathrm{~m}^{3}$ of material discharged, c last stage of discharge defining the remaining material on silo

stages. For $1 \mathrm{~m}^{3}$ of discharged volume, particles adjacent to the outlet are in motion, followed by particles further afield. A velocity wave propagates upward and the funnel flow behavior is clearly initiated. Ore pellets located toward the wall remain stagnant-the continuous lines, at least for the five lower levels, remain horizontal at a significant distance from the wall-, indicating that the material does not crumbles toward the outlet; the material that is discharged is the one located at the center of the silo.

The definition of the funnel flow is more pronounced as the material is discharged; for $5 \mathrm{~m}^{3}$ only the material located at the center of the silo of the last two levels remains in the silo, and for $17 \mathrm{~m}^{3}$ the funnel is developed completely. This can be seen by the clearly definition of two principal zones:

Fig. 3 Flow patterns comparison between experiments and PFEM for different volumes of discharge: a $1 \mathrm{~m}^{3}$, b $5 \mathrm{~m}^{3}$, c $17 \mathrm{~m}^{3}$

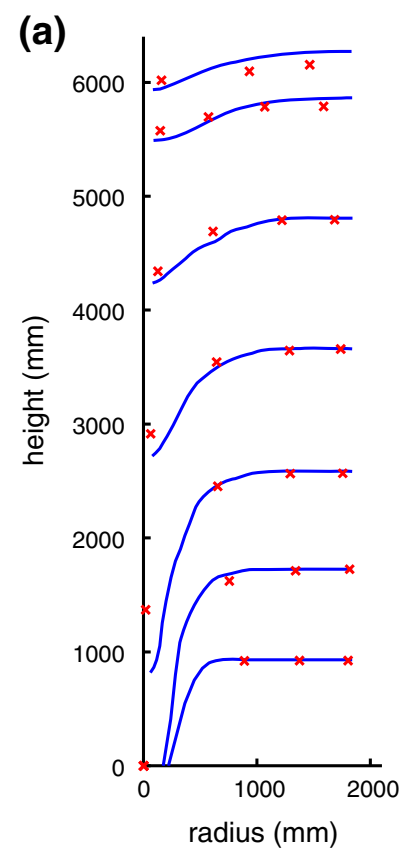

a stagnant region located toward the silo walls and the flow region defined by a loose material above the outlet of the silo.

Nonetheless, significant differences with computer visualization data are observed, as we detail in what follows. For $5 \mathrm{~m}^{3}$ of discharge, the two blue lines (counting from the top) exhibit a separation between them and a small horizontal platform toward the wall is still apparent; by contrast, the plot of the corresponding experimental tags defines a unique overlapped contour level. The material volume defined by the skyline of the tags seems less than the one defined by the numerical result, however the experimental volume discharged is $5 \mathrm{~m}^{3}$. The progressive decrease of volume observed experimentally at $17 \mathrm{~m}^{3}$ of discharge is more pronounced that the decrease in volume predicted by the model, which even retain horizontal platforms of stagnant material. This markedly distinct response highlights concern with the characterization of the material response, which should clearly receive careful consideration in future improvements of the model.

These differences between observed and calculated flow pattern responses may be attributed to three main reasons. The first one is the numerous simplifying assumptions made in Sect. 2 in deriving the constitutive model, the second reason due to assumptions introduced to be able to do the simulation, and the third reason concerns the computer visualization code required to visualize the position of the tags. Among the simplifying assumptions made in the model, we suppose that the internal friction coefficient is independent of the pressure or of the material density. A similar comment can be done on other parameters like the viscosity (defined in terms of the relaxation time).

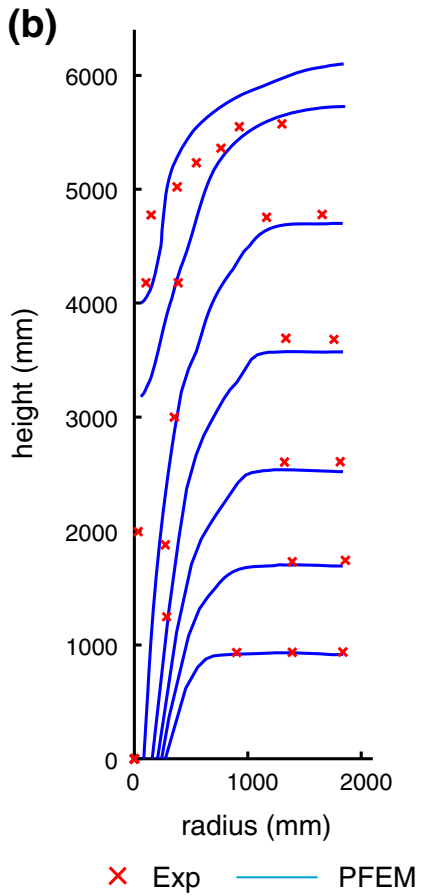



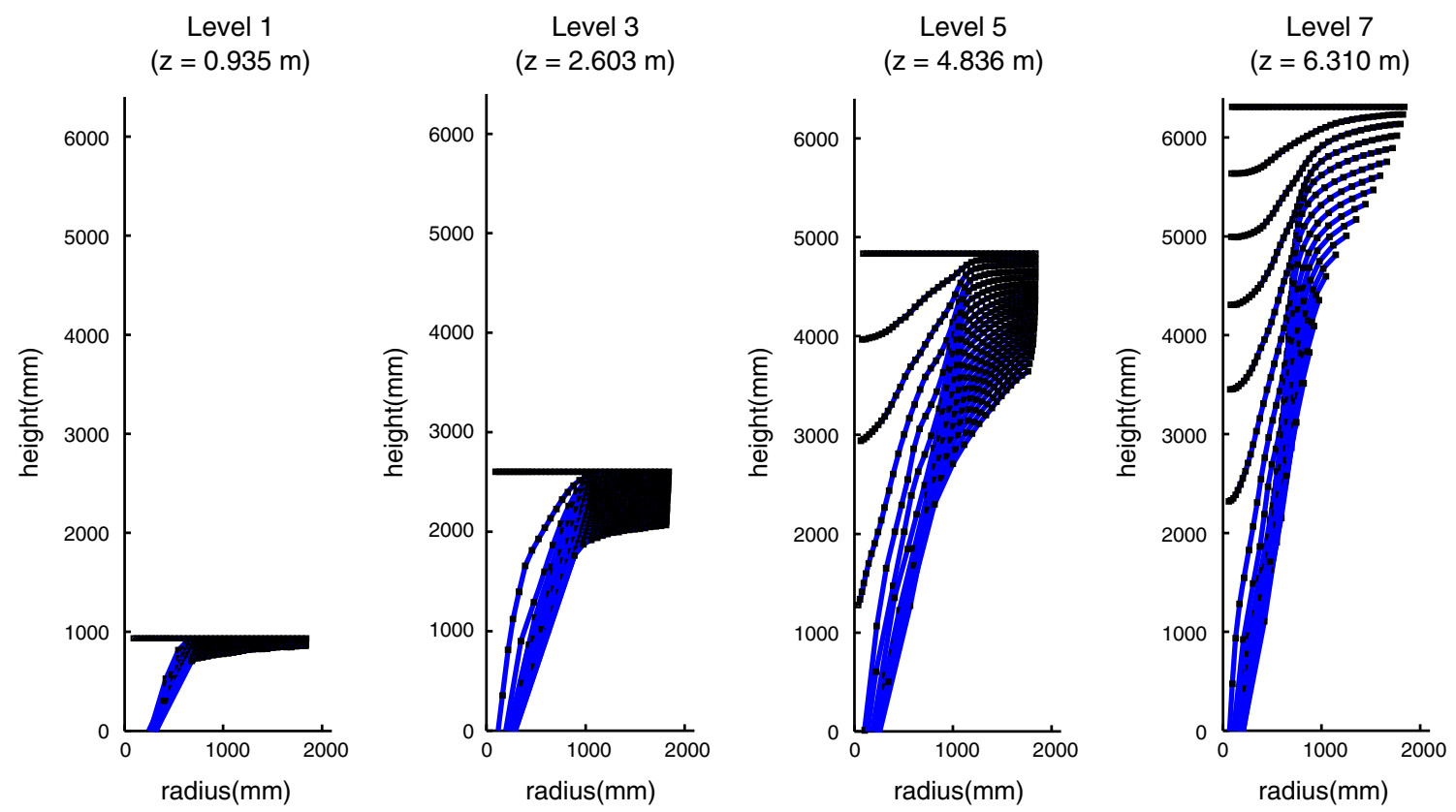

Fig. 4 Evolution of the position for different levels during the material discharge

Another contributor to these discrepancies may be found in the elementary character of the outlet's size and shape. As commented in Sect. 3.1, the outlet has a hydraulically controlled slide gate, which is opened partially, making the outlet in a shape of a circular segment; by contrast, in order to minimize the computational cost, we assume an axisymmetric geometry, removing in this way the intrinsic $3 \mathrm{D}$ character of the outlet. The third reason is the computer visualization code itself. As pointed out by the authors of the experiment in [4], the interpretations of the residence time measurements were made following previous studies by portraying residence times in horizontal and vertical cross-sections through the silo. These contours are more as a qualitative indicator of the flow pattern than a quantitative one.

An alternative numerical representation of the flow patterns is discussed in what follows. The idea is to plot in the same domain the evolution of a fixed material line. In order to get a clear visualization only a few time steps are included in the analysis. The different positions of the odd levels (counting from the bottom) have been drawn in Fig. 4. There are plotted several particles at each level identified by black dots and joined by a continuous blue line; the material position of the particles at different time steps defines the flow mechanisms present during its discharge.

It is identified a similar pattern of discharge on all the levels which define the evolution of a funnel flow with a small contribution of a mass pattern. The particles located close to the wall do not present a noticeable separation in their relative position, but, close to the outlet, they change abruptly as they converge on the flow. The discharge mechanism is not considered fully of the funnel type, since it is identified that the material located toward the wall is not completely stagnant, i.e. it is observed that the particles diminish their level, indicating a discharge of some material under them.

\subsubsection{Influence of material and geometric parameters on the flow patterns}

The advantage of numerical simulations is the capability of easily varying parameters in order to have a wider understanding of the phenomenon; in this section it is studied the influence of the material and geometric parameters on the discharge mechanisms of a silo.

The first set of examples corresponds to the study of the flow patterns varying the internal friction coefficient. In order to identify a proper variation discharge mechanism due to this coefficient, it was defined a set of values above and under the reference one; this material coefficient was varied using the following set $b_{1}=[0.7,0.85,1,1.15]$ - the calibrated coefficient with respect the experimental essay is $b_{1}=1.00$.

The results of this study are summarized in Fig. 5, where the flow patterns for the set of internal friction coefficients are compared.

In the figure are plotted, in a front view with a continuous blue line, the position of the markers at each level for different volumes of material discharged-1, 5, 10, 15, and $20 \mathrm{~m}^{3}$; with a dotted red line, it is marked the original height for the second and fourth levels, to observe its variation during the discharge. The front views show for the four cases, from left to right, how the flow evolves since the material is released. 

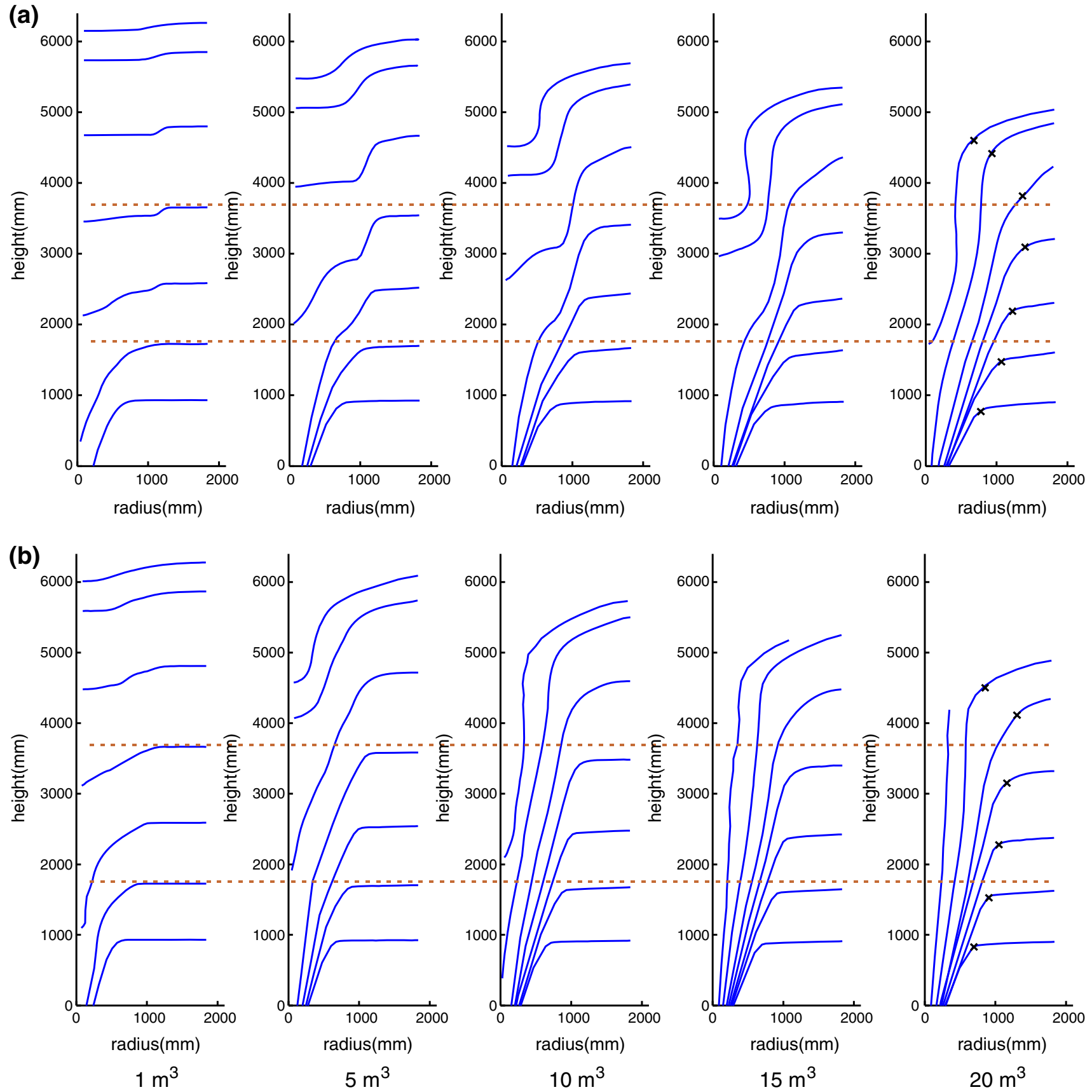

Fig. 5 Variation of level markers during material discharge-1, 5, 10, 15, and $20 \mathrm{~m}^{3}$-for different internal friction angles: a $b_{1}=0.70$, b $b_{1}=0.85$, c $b_{1}=1.00$, and $\mathbf{d} b_{1}=1.15$

In the four cases, the flow is identified as being of the funnel type; it is observed on the first figure, $1 \mathrm{~m}^{3}$, that the flow is formed at the center or axisymmetric axes of the silo. As the material continue to be discharged, the stagnant zones are more clearly identified-shown at the fourth and fifth figures of each internal friction coefficient, corresponding to 15 and $20 \mathrm{~m}^{3}$ of material discharged.

As it is expected, the internal friction coefficient of the model plays a fundamental role in the behavior of the mate- rial. Lower values of internal friction coefficient allow the material to develop larger deformations for the same external excitation-in this case gravitational forces. Figure 5(a) shows a funnel flow with a high contribution of mass flow during the discharge, which is described by a flow nourished by material located of the bottom region of the silo. The lower levels of markers show a narrow region of stagnant material toward the wall, which describes a funnel flow. The mass flow contribution is identified by the large amount of mate- 

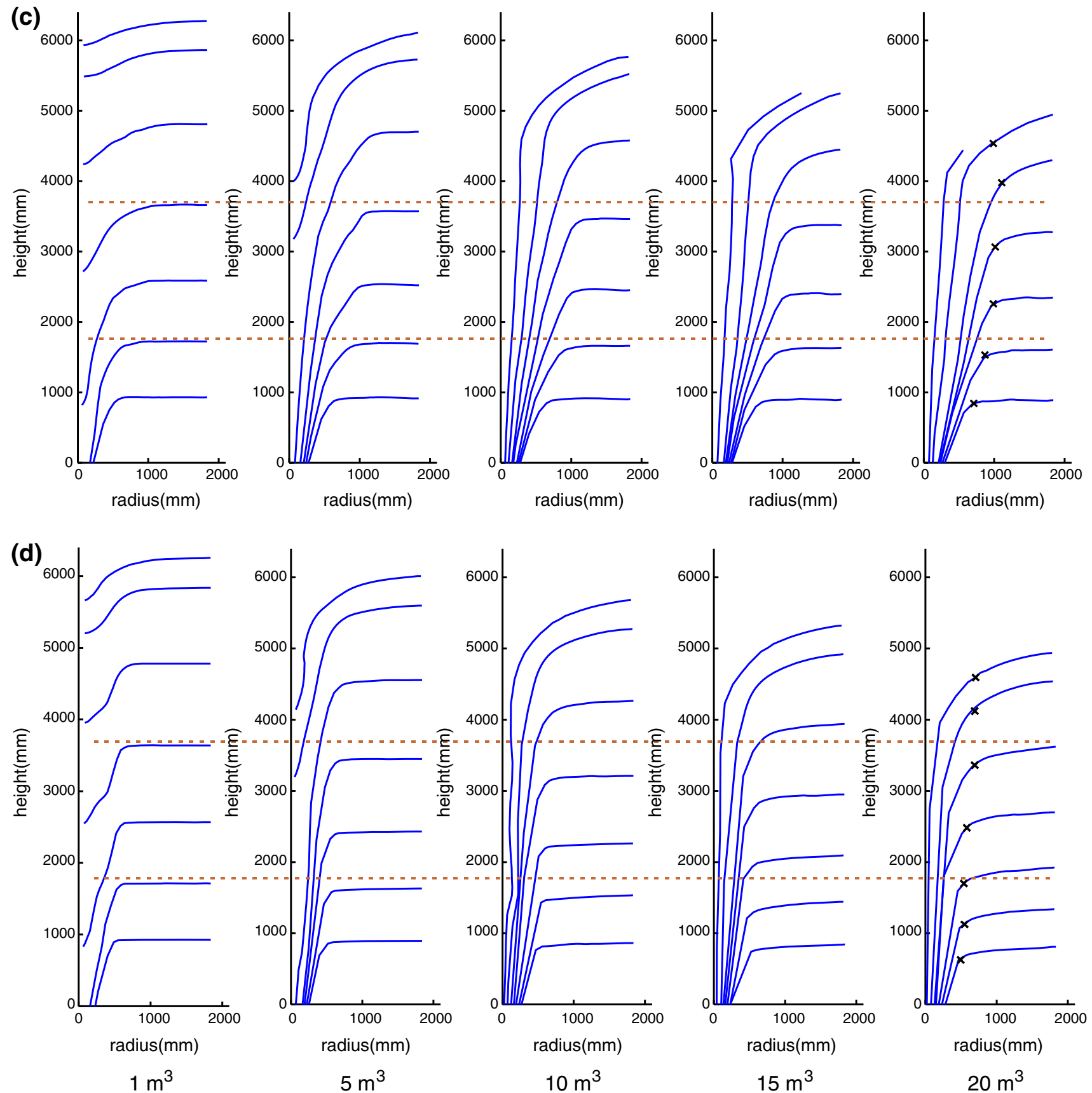

Fig. 5 continued

rial discharged from the bottom of the silo since the upper tags markers remain on the silo-level 6 and 7 for $15 \mathrm{~m}^{3}$ and level 7 for $20 \mathrm{~m}^{3}$ of material discharged.

The funnel flow is recovered with a slight increment on the internal friction coefficient. Figure 5(b) displays the flow patterns obtained for a discharge using $b_{1}=0.85$. It is observed a wider region of the stagnant zone, releasing material of the upper levels. Comparing with previous internal friction coefficient, it is observed that the uppermost level has been released at $15 \mathrm{~m}^{3}$ of material discharged.
The flow patterns corresponding to the reference internal friction coefficient are plotted in Fig. 5(c). The funnel is achieved with the discharge of $15 \mathrm{~m}^{3}$ of material - material from the last level has been discharged; and the stagnant zones are wider as expected. A higher value than the reference example was used for the internal friction coefficient, in order to force a more pronounced flow funnel. Figure 5(d) shows the flow patterns for a value of $b_{1}=1.15$ (corresponding to a Mohr-Coulomb internal friction angle $\theta \approx 34.77^{\circ}$ ). As the internal friction coefficient is increased, the stagnant 
zone is increased toward the centre of the silo defining a vertical narrow region of flow closer to the axisymmetric axis. Since the funnel flow is more pronounced, the material corresponding to the upper levels of the marker is released since the first $10 \mathrm{~m}^{3}$ of material discharged.

The influence of the internal friction coefficient on the discharge mechanisms could be summarized studying the flow patterns present at $20 \mathrm{~m}^{3}$ for each friction coefficient value. It was marked with a black cross mark at the inflection point of the curvature of each level of the markers. It is identified that the flow encompasses a wider region as the internal friction coefficient is smaller; for $b_{1}=0.7$ the loosen material present an elliptic region, while, for a granular media with $b_{1}=1.15$, the loosen material flow is defined in a narrow vertical region.

The second study consists of the analysis of the opening size of the outlet on the discharge of the silo. From the previous set of examples, it is identified a strong influence on the flow type due to the internal friction coefficient; even though, it is indispensable to study the dependency of the flow as the dimensions of the outlet are modified. The study analyzes the flow patterns during discharge for three different outlet sizes and constraining the value of the internal friction coefficient with the reference example $b_{1}=1$. The radii to explore, for the full-circular outlet, are: 170, 240, and 310 $\mathrm{mm}$ - the outlet radius used in the reference example shown in the previous section is $240 \mathrm{~mm}$, the corresponding flow patterns were plotted in Fig. 5(c).

Figure 6 compares the variation of the level markers as a function of the flow type for the remaining two radii at 1 , $5,10,15$, and $20 \mathrm{~m}^{3}$. The continuous blue line denotes the evolution of seven levels of the markers, and the dotted red lines are used, as in previous figure, as a reference for the height of the second and fourth levels. It is observed that, for both discharges, the predominant flow is of funnel type. Even though, the patterns show a variation on the definition of the stagnant zones and the flow region on the silo.

Figure 6(a) shows the evolution of level markers for the smallest radius used for the outlets; the flow patterns show a broader stagnant zone than for larger radius, see Figs. 5(c) and 6(b) for $R=240$ and $310 \mathrm{~mm}$ respectively. The flow region is defined by a vertical narrow region located at the center of the silo; although it is observed a delay on the discharge of the upper levels - the material on these levels remains inside the silo after $20 \mathrm{~m}^{3}$ of material discharged.

The largest radius of the outlet shows likewise a semimass flow discharge. In contrast with the smaller outlets, the core of the funnel flow spreads toward the walls on the upper levels of the silo. This reduction of the stagnant zone on the upper levels appears even at the first stages of the discharge ( 1 and $5 \mathrm{~m}^{3}$ of discharged material in Fig. 6b).

\subsubsection{Velocity fields during silo discharge}

Velocity fields on the silo are information required to determine design parameters, as the residence time distribution, mixing properties of the material, and the rate of wall wear [2]. Numerical simulations allow processing the data accordingly to understand different features of the phenomenon. In this section, the velocity contours obtained for different amounts of material discharged and the maximum velocities obtained during the whole process are discussed.

Plotting the contours of the velocity field is an alternative procedure to understand the discharge mechanisms on a silo. In this context, the contours corresponding to different volumes of discharged material, internal friction coefficient $b_{1}=1$ and an the outlet radius $R=170 \mathrm{~mm}$, are shown in Fig. 7. There, it can be observed that the funnel flow develops since the first cubic meters of material are discharged.

The transition between a stagnant zone and a flow zone is defined by the presence of a discontinuity on the velocities. This discontinuity is not necessary be coincident with a stress discontinuity since it could be present in a continuous stress field [2]. The term discontinuity for a velocity field is not as strict as in the stress field; in terms of a velocity field, it is defined by a large velocity gradient over a small distance.

The velocity contours, shown in Fig. 7, allow characterizing this discontinuity. Particles moving with a velocity greater than $0.10 \mathrm{~m} / \mathrm{s}$ are represented by the dark color, and particles moving with a velocity lower than $0.1 \mathrm{~m} / \mathrm{s}$ are represented by the light color. This technique is used to provide a qualitative illustration of the developing flow pattern by differentiating between zones that are moving relative to each other.

Velocity contours supplement the understanding of the granular flow. It can be observed, from the previous figure, that the discharge is characterized by a vertical sliding of a rigid block of material-nourished by the material from the upper levels - and that, after a certain distance from the outlet, the material is loosen and the particles gain velocity toward the outlet.

The maximum velocities appearing during the whole discharge allow identifying the smoothness of the flow during the process. Figure 8 shows the comparison of the maximum velocities reached during the discharge of two models-for the outlet dimension $R=170$ and $240 \mathrm{~mm}$. From those curves two main features are identified: the discharge of the material takes place in a steady state regime during the full process; and second, as it is expected, the maximum velocities on the material are increased as the silo's outlet radius is larger. 

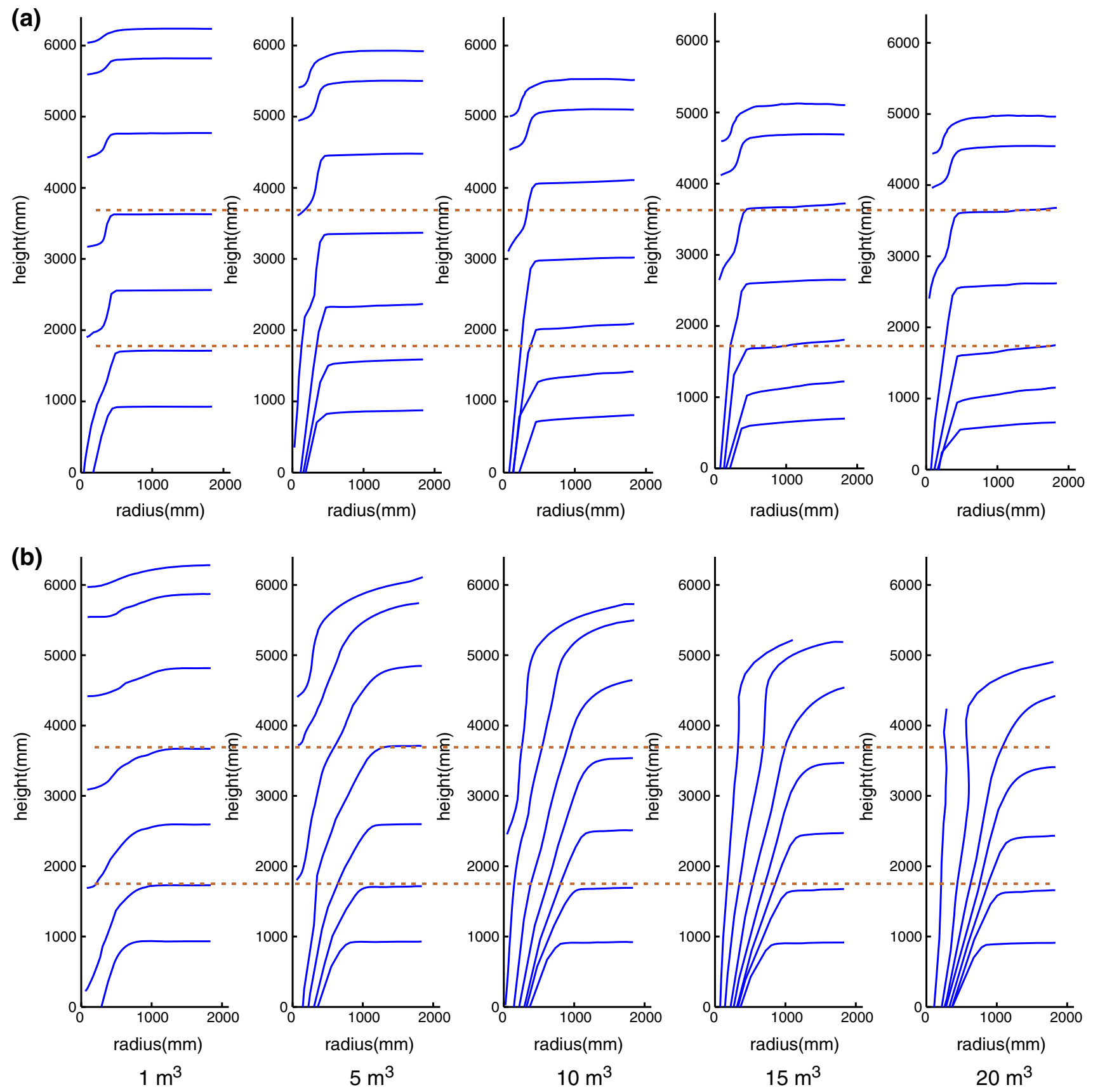

Fig. 6 Variation of level markers during material discharge—discharge at 1, 5, 10, 15, and $20 \mathrm{~m}^{3}$ — for different outlet radius: a $R=170 \mathrm{~mm}$, b $R=310 \mathrm{~mm}$

\subsubsection{Pressure distribution}

Probably the most relevant result from the analysis is the pressure distribution acting on the silo wall. Unfortunately, wall pressures for this experimental test are not reported on the referenced technical report [33] since measurement difficulties appeared during the experiments. Nevertheless, numerical pressure distributions can be analyzed from the previous validation campaign of the flow patterns.
It is expected that numerical pressures distribution differ from hydrostatic distributions in a static condition. These differences result due to the influence of the internal friction angle and wall friction, which the last, gradually transfers vertical loads into the walls.

Firstly, we reproduce numerically the hydrostatic analysis, in which the silo is hypothetical filled with water and the resulting numerical pressure is compared with the hydrostatic distribution. In order to reproduce the mechanical behavior of 
(a)

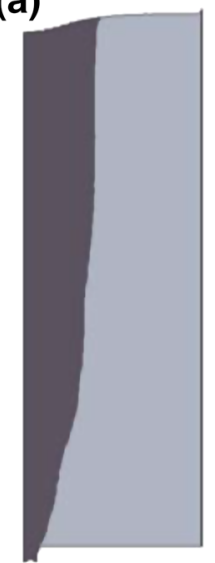

(b)

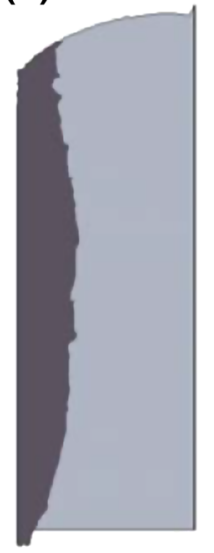

(c)

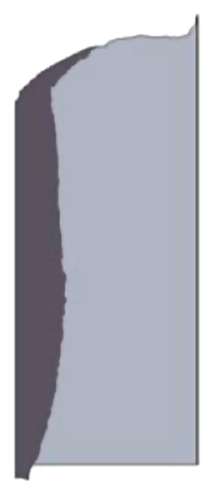

Fig. 7 Velocity contours for material with internal friction coefficient $b_{1}=1$ through an outlet with radius $R=170 \mathrm{~mm}: \mathbf{a} 1 \mathrm{~m}^{3}, \mathbf{b ~} 5 \mathrm{~m}^{3}$, and $\mathbf{c} 20 \mathrm{~m}^{3}$. Particles moving with a velocity greater than $0.1 \mathrm{~m} / \mathrm{s}$ are represented by dark color, and particles moving with a velocity lower than $0.1 \mathrm{~m} / \mathrm{s}$ are represented by light color

the fluid by means of our constitutive model, the internal friction coefficient of the material was set close to zero, $b_{1} \approx 0$. Figure 9 (a) shows the wall pressure distribution for different bulk modulus compared with the hydrostatic pressure distribution. The difference between the curves is a consequence of the compressibility of the elastic part of the model and the theoretical hydrostatic pressure is recovered when the bulk modulus is increased (see Fig. 9a). Our concern now is to examine the response of the numerical results when the internal friction coefficient is increased. These results are shown in Fig. 9(b). It is observed that the maximum wall pressures are reduced as the internal friction is increased.

Likewise, the pressures reached in the silo's wall and the granular material are depending on the geometry of the problem itself; Fig. 10 presents the pressures for different volumes of material discharged using two different opening size on their outlets. Figure 10(a.1) shows the pressure evolution on the material for an outlet radius $R=170 \mathrm{~mm}$; it is observed that the stresses fields suffer a redistribution when the mate-
Fig. 8 Maximum velocities for a material with internal friction angle $b_{1}=1$ comparing two different outlets $-R=170 \mathrm{~mm}$ and $R=240 \mathrm{~mm}$
Fig. 9 Wall pressures when material confined: a wall pressure distribution for different bulk modulus compared with the hydrostatic pressure; $\mathbf{b}$ variation of pressure distributions on the silo's wall for different internal friction coefficients

Fig. 10 Evolution of pressures distributions during the discharge of a: a Silo with an outlet of $R=170 \mathrm{~mm}$, b Silo with an outlet of $R=240 \mathrm{~mm}$. It is displayed first the material pressures, then wall pressures exerted by the material
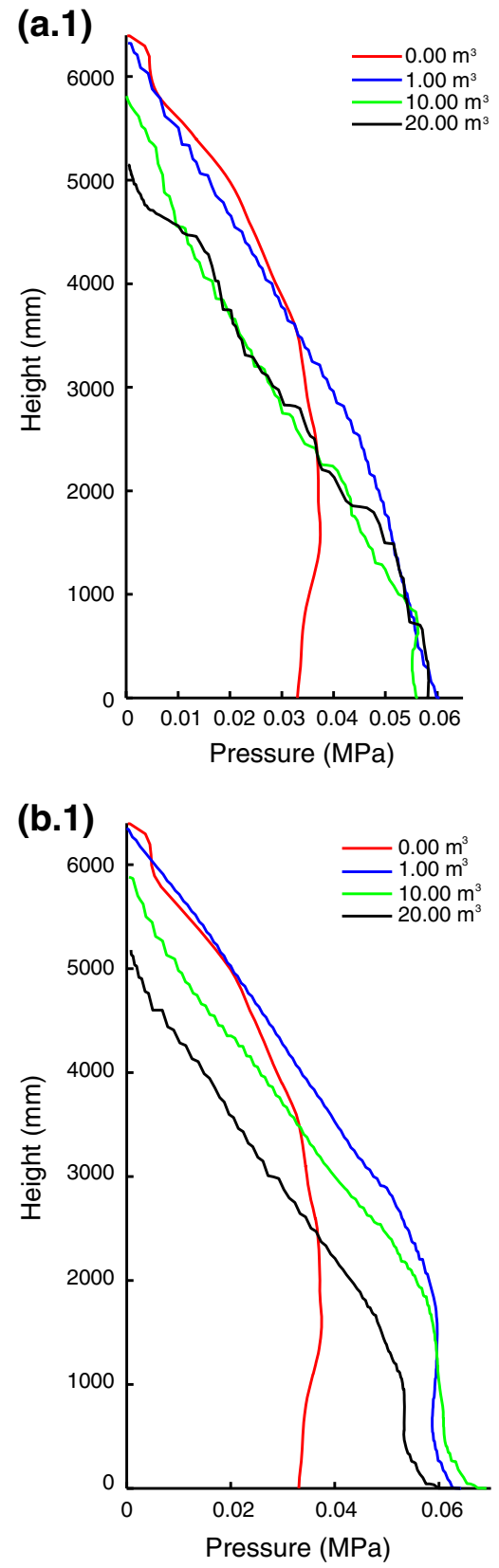


rial is released. The pressures on the material located at the wall are increased up to $50 \%$ just as the outlet is opened-as it is displayed by the variations on the values for a material confined and at $1 \mathrm{~m}^{3}$ of material discharged. In contrast with the material pressures, the wall pressures do not suffer a substantial increment when the material is released, see Fig. 10(a.2). In this case, the radial stress is incremented at the bottom of the silo and reduced almost linearly toward its upper level.

For both properties, material pressure and wall pressure, the maximum values are present at the bottom of the silo. During the discharge, pressures along the wall are reduced, since there is a lower amount of material inside the silo, but the maximum values at the bottom remain constant. This monotonic behavior is expected, since it corresponds to a concentric discharge-maximum values for the highest amount of material in the silo, which decrease as it is discharged.

The evolution of the stresses during the material discharge is similar for the outlet of radius $R=240 \mathrm{~mm}$. Figure 10(b.1) shows the material pressures for different amounts of discharge; the comparison with those obtained with the smaller outlet shows that the mechanical response of the material is similar, presenting an increment up to $50 \%$ of the magnitude on the pressures at the bottom once the outlet is opened. The difference on the pressure redistribution between both 
Fig. 11 Scaled model of a tumbling ball mill, all measures in mm: a Front view, $\mathbf{b}$ model depth [9]
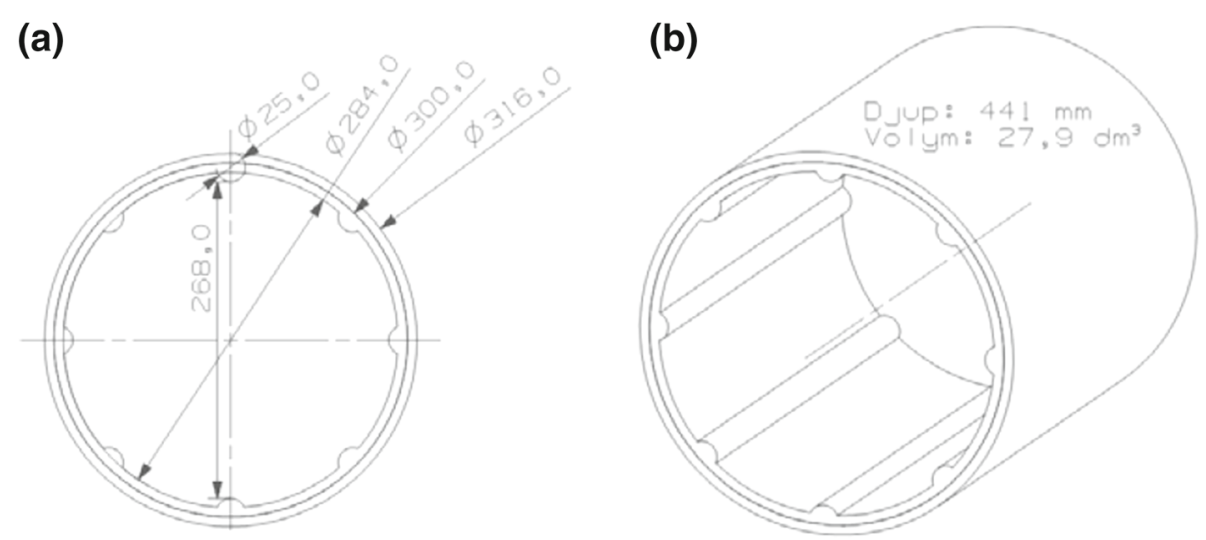

Fig. 12 Averaged power measured on dry sand [9]

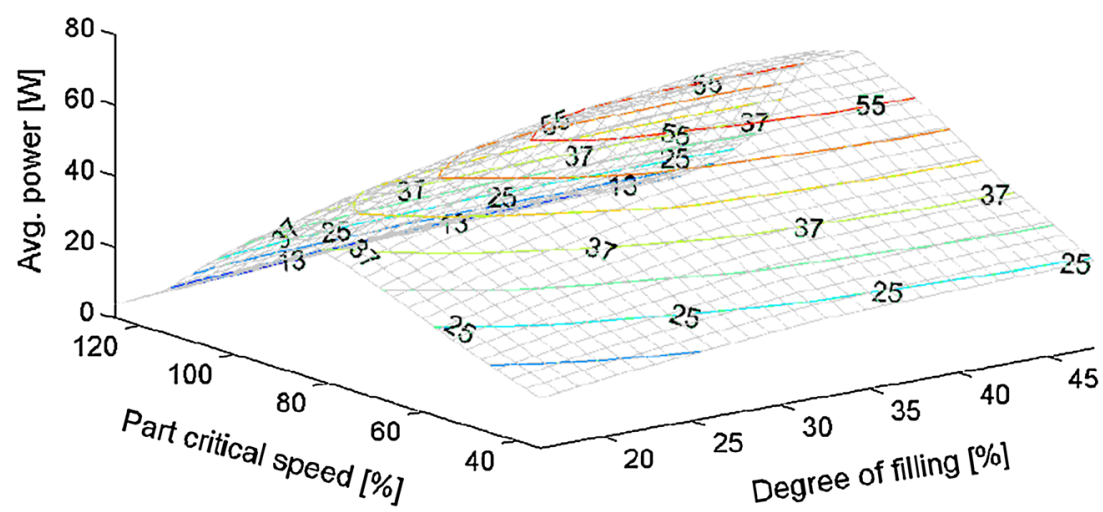

outlets size cases, is that, for the wider opening, the level of pressures on the material near the wall maintains the same magnitude - almost for the two first meters from the bottom of the material.

The pressures exerted by the material on the wall, for different volumes of discharge, are shown in Fig. 10(b.2). The evolution of the pressures shows the same behavior than for the smaller outlet, being the only difference a slightly increment on the magnitude of the pressures. Since the wall pressures for both discharges show a similar response, it is expected that the increment on the material pressures be caused by a redistribution of the gravitational loads of the material.

\subsection{Experimental measurement of power consumption for a tumbling mill—experimental setup}

The model capability to simulate tumbling processes is verified via the numerical simulation of the experimental tests conducted in [9]. The experiment consists of the measurement of power consumption during the comminution of a mill charge, varying the granular mass and the rotational velocity. The measurements were conducted on a laboratory-scale ball mill. The scaled model has an inner diameter $\phi_{i}=284 \mathrm{~mm}$ and a depth $l=441 \mathrm{~mm}$; the displacement of the charge is controlled by eight semi- circular bumps of diameter $\phi_{b}=25 \mathrm{~mm}$ spaced uniformly as lifters. The charge consists of dry sand of density about $2,500 \mathrm{~kg} / \mathrm{m}^{3}$ and porosity around $33 \%$. Figure 11 shows the drum dimensions as well as the placement of the lifters.

The computation of the power is given in terms of the torque applied to the drum and its angular speed. The torque was measured as the reaction force applied on a load cell at a given distance from the rotation center, and the average rotational speed of the system was obtained measuring the number of revolutions during a long time. Due to the impulsive nature of the charge, it is convenient to measure the average torque and the angular speed, to define a relatively invariant measure of the required power [35].

The rotational speed at which the drum was subjected to, is defined in terms of a critical speed of the system, given by the following equation

$\omega_{\text {crit }}=60 \frac{\sqrt{g / r_{i}}}{2 \pi}$

where $g$ is the acceleration of gravity, $r_{i}$ the inner radius, and $\omega_{\text {crit }}$ the speed given in rpm. Figure 12 shows the averaged measured power for the system correlated to the degree of filling of the drum and its critical speed. 
Fig. 13 Velocity contours for the granular flow $(\mathrm{m} / \mathrm{s})$ : $\mathbf{a}$ transient regime, acceleration process, $\mathbf{b}$ stationary regime, after two revolutions (a)

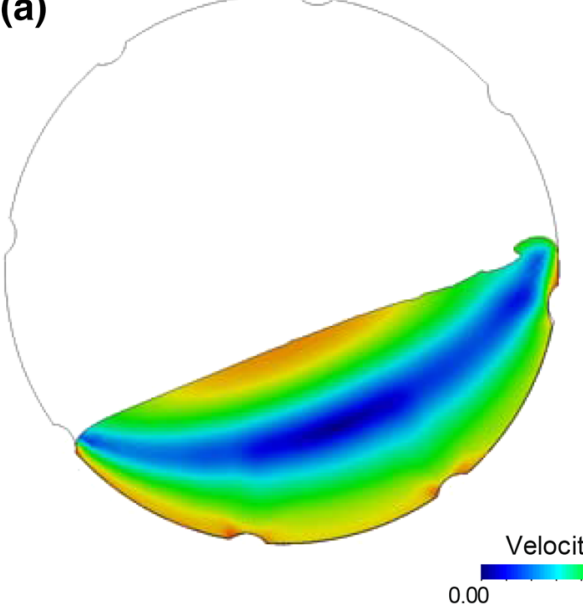

(b)

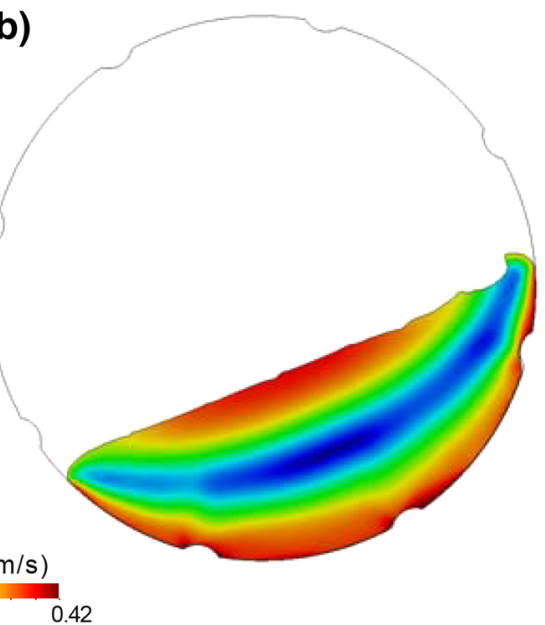

\subsection{Numerical simulations of a tumbling ball mill}

As mentioned earlier, the proposed constitutive model is, in principle, only suited for representing dry dense granular materials. In the present example (tumbling ball mill), the drum reaches relatively high rotational speeds and the granular material tend to separate. The assumption of dense flow, thus, is not strictly valid in the context of this example, and, therefore, an accurate representation of the motion of the material within the drum cannot be expected. However, our interest does not lay on an accurate simulation of such local details, but rather on examining the overall behavior of the system in terms of the mechanical power required to maintain the tumbling process. Accordingly, numerical and experimental torque values will be compared for three different rotational speeds - 35,65 , and $95 \%$ of the system critical speed. The smallest rotational speed will be used to calibrate the internal friction coefficient and the relaxation time $\left(b_{1}\right.$ and $\tau_{R}$, respectively), while the other two cases will be used for validation.

The degree of filling of the drum is $35 \%$. The geometry of the model corresponds directly to the experimental setup using an internal diameter of $\phi_{i}=284 \mathrm{~mm}$, and eight semicircular bumps of diameter $\phi_{b}=25 \mathrm{~mm}$ as the lifters of the mill. The experimental drum is modelled using a twodimensional plain-strain state of depth $l=441 \mathrm{~mm}$. The initial spatial discretization has approximately 2,250 nodes and 4,500 linear triangular elements. As regards time discretization, time steps of $\Delta t=1 / 10,000 \mathrm{~s}$. are used. The granular material $/$ mill structure plate interaction is modelled with the same algorithm used for the silo example; in this case the wall friction coefficient is set to the same value than in the bulk domain.

As for the material properties of the granular material, the internal friction coefficient and the wall friction coefficient is set to $b_{1}=0.30$, and the cohesion parameter to $b_{2}=0.01 \mathrm{~Pa}$ (this relatively small value of cohesion is introduced to ensure numerical stability). Likewise, the employed bulk and shear modulus are $\kappa=32 \mathrm{MPa}$ and $G=7 \mathrm{MPa}$, respectively. The visco-plastic regularization is a function of the relaxation time $\tau_{R}$; for these tests, the relaxation time is $\tau_{R}=5 \times 10^{-6} \mathrm{~s}$.

In order to calculate the power required by the mill to keep its rotational speed, the balance of the mechanical energy of the system is used. This balance states that the sum of the rate of change of the kinetic energy $\kappa_{e}$ and the rate of the internal mechanical work, $P_{\text {int }}$, of a continuum is equal to the rate of external mechanical work $P_{\text {ext }}$ [36], as given by the following equation

$$
\frac{D}{D t} \kappa_{e}(t)+P_{\mathrm{int}}(t)=P_{\mathrm{ext}}(t)
$$

Since the kinetic energy is a function of the velocity field and the internal mechanical work is a function of the stress field, it is fundamental to obtain a smooth response of those fields. The velocity contours, shown in Fig. 13, determine the kinematic response of the charge while the drum rotates. This velocities distribution presents the same pattern in the transient and stationary regime, varying the magnitudes reached in each stage.

The motion of the charge is defined by an overturn of the material from the wall toward the center, where the larger velocities show up at the outer region of the material and relative null velocities are located on the core of the material. A relevant difference between the transient and stationary regimes is that, on its acceleration stage the material presents a higher degree of consolidation, see Fig. 13(a). Instead, when the material reaches the stationary regime, Fig. 13(b), it is observed that the material experiences a relaxation.

Pressure contours are displayed in Fig. 14. It is worth noting that computed pressures are relatively smooth, a fact that indicates that the used mixed formulation is fulfilling the purpose of overcoming the deleterious effects of volumetric locking. Likewise, it can be observed that pressures in the tensile regime are, at least, one order of magnitude smaller than 
Fig. 14 Pressure distribution on the material (MPa): a material at rest, $\mathbf{b}$ material at stationary regime after two revolutions at constant velocity

(a)

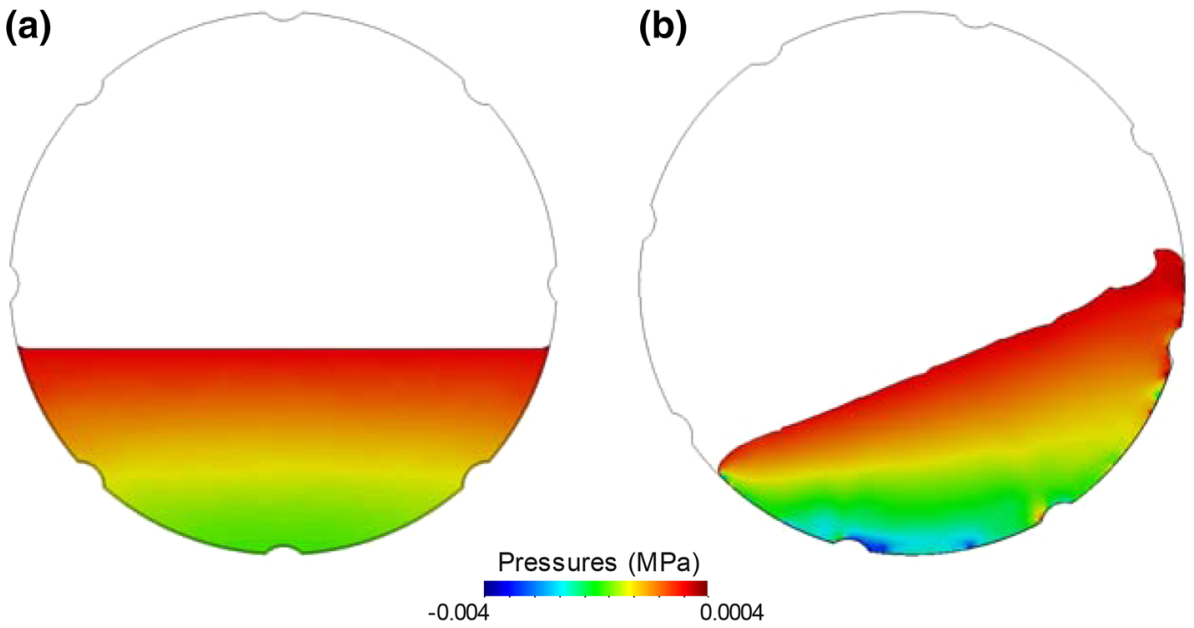

Fig. 15 Torque evolution-transient and stationary response

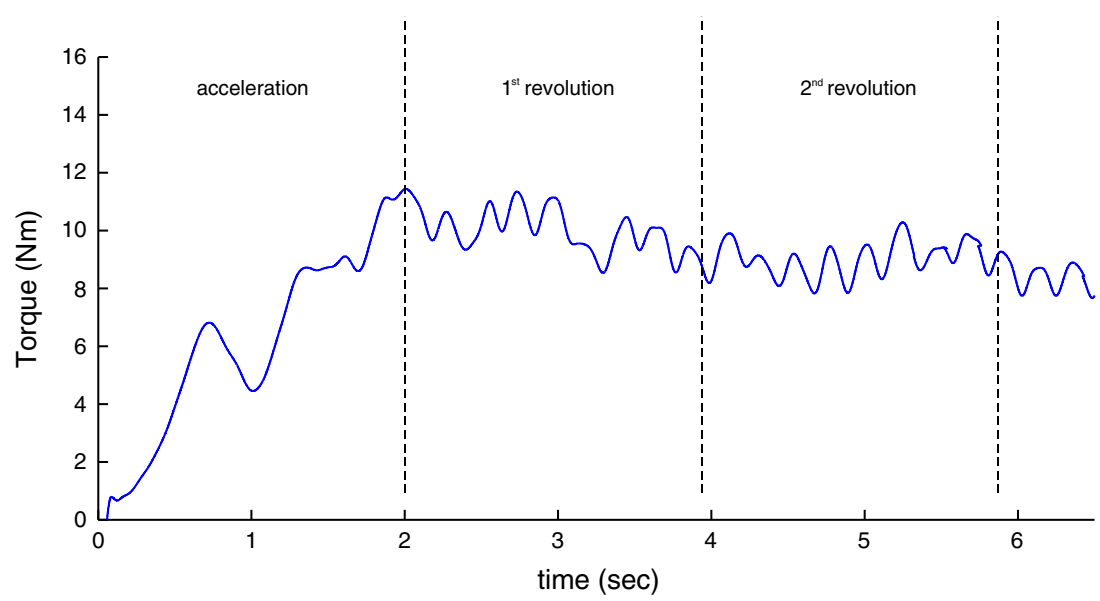

those in the compressive regime. The contours indicate that the maximum compressive pressures are given on the charge propelled by the bump or lifter, and that, after the material overpass the bump, a region of loose material is formed presenting small or null compressive pressures, see Fig. 14(b).

The torque obtained from the numerical simulations is computed using the internal power defined in Eq. (21), and the rotational speed of the system. The experimental torque data reported corresponds to a full revolution in its steady state. Therefore, in order to compare with numerical simulations, it is required to measure the torque in a steady state. The loading conditions for the tumbling mill are defined by an acceleration period of two seconds until the rotational speed is reached; thereafter, it is set a complete revolution to reach the steady regime at the second revolution with constant velocity, which is the one to be compared.

Figure 15 shows the evolution of the torque curve of the numerical simulation, for a rotational speed at $35 \%$ of the critical speed, during the three loading stages-an acceleration period of two seconds, a revolution on transient regime, and the stationary regime reached at the second revolution. The maximum value of the torque is reached at the transition between the acceleration stage and the condition of constant rotational speed. It is observed a slight diminution of the torque value on the first revolution. This is due to the relaxation that the material suffers until it reaches its steady state.

Comparison between experimental and numerical torque for different rotational speeds is shown in Fig. 16. For the experimental data, a continuous red line is used. Numerical values, on the other hand, are represented by a continuous black line. The comparison shows the values for a full revolution of the drum, once the steady state is reached.

Figure 16(a) shows the torque comparison for a rotational speed corresponding to $35 \%$ of its critical speed, which is the one used for calibration purposes. While a reasonable overall agreement is achieved in terms of mean response, discrepancies are detected in the oscillating pattern around this mean value. Indeed, experimental torque oscillates more abruptly, and at a higher frequency, than its numerical counterpart does. It can be easily shown that the period of the oscillations in the numerical results is related with the circumferential spacing of the bumps in the drum.

The comparison with experimental data for higher velocities presents the same behavior than in the above commented 
Fig. 16 Torque comparison between experimental and numerical models for rotational speeds at: a $35 \%$ of critical speed, b $65 \%$ of critical speed, c $95 \%$ of critical speed
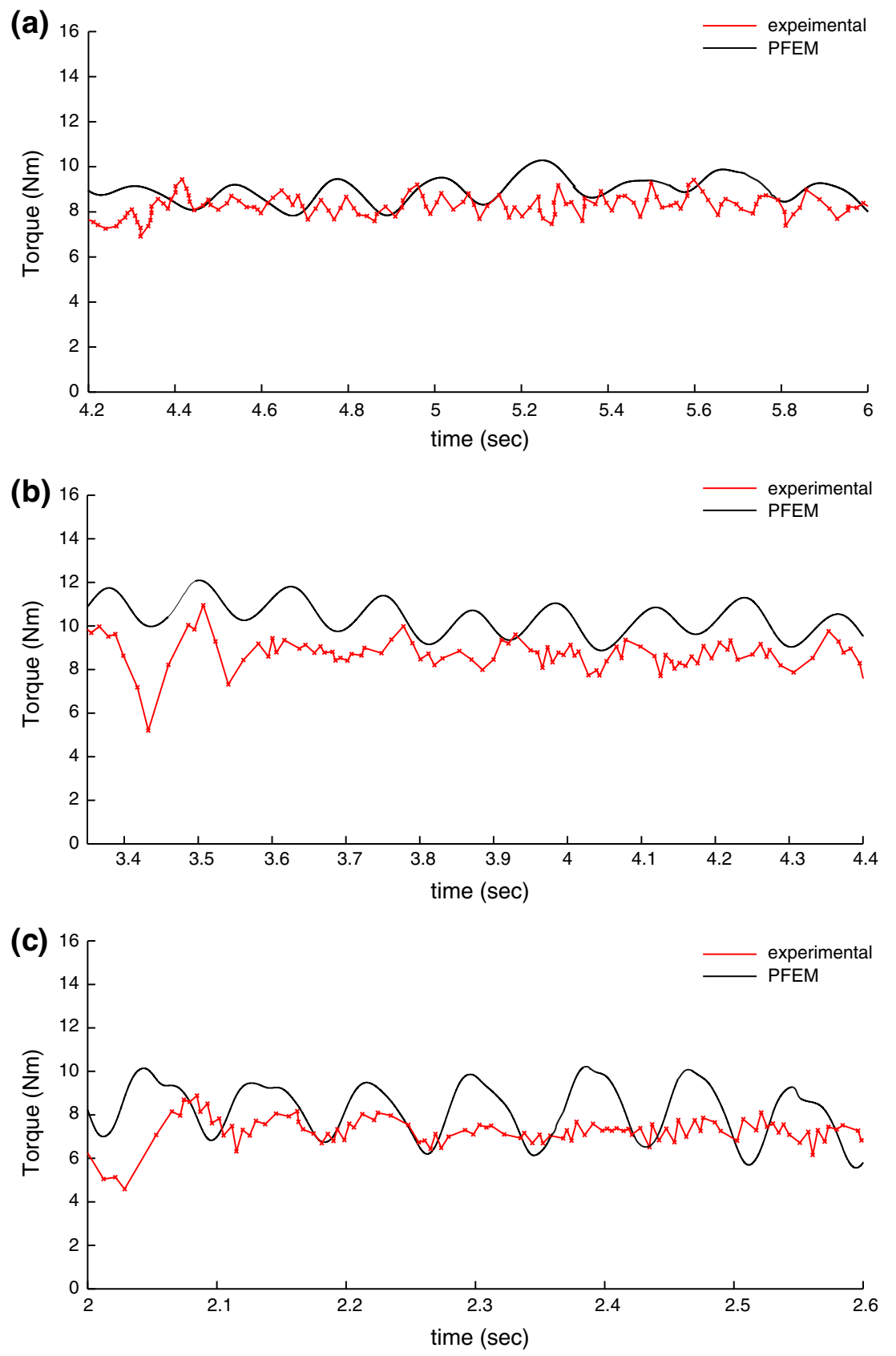

reference example. Figure 16(b) shows the comparison for a rotational speed at $65 \%$ of the critical speed. The torque presents, as in previous example, an oscillatory response that corresponds to the interaction of the material with the bumps of the drum. It is remarked that the amplitude of the oscillations in the computed results are notably higher than in the previous case.

Lastly, the torque for a rotational speed of $95 \%$ is given in Fig. 16(c). The same oscillation pattern is observed, with a further increase in amplitude. This fact confirms the influence of the rotational speed in the amplitude of the oscillations predicted by the numerical model. This influence, however, is not perceived in the experimental graphs (the amplitude in this case is, on average, similar in the three studied cases). Nevertheless, the model is able to capture with reasonable accuracy the mean torque in the three cases. This is confirmed by the comparison of averaged power of the experimental data and the one obtained by numerical simulation for the three different rotational speeds, see Fig. 17. As expected, the highest accuracy is observed for the lowest rotational speed, for this is the one used to calibrate the model. As the speed is increased, the deviation between experimental and numerical results increases, albeit it remains at moderate levels. In conclusion, despite the limitation of the model, the 
Fig. 17 Average power comparison for the three different rotational speeds

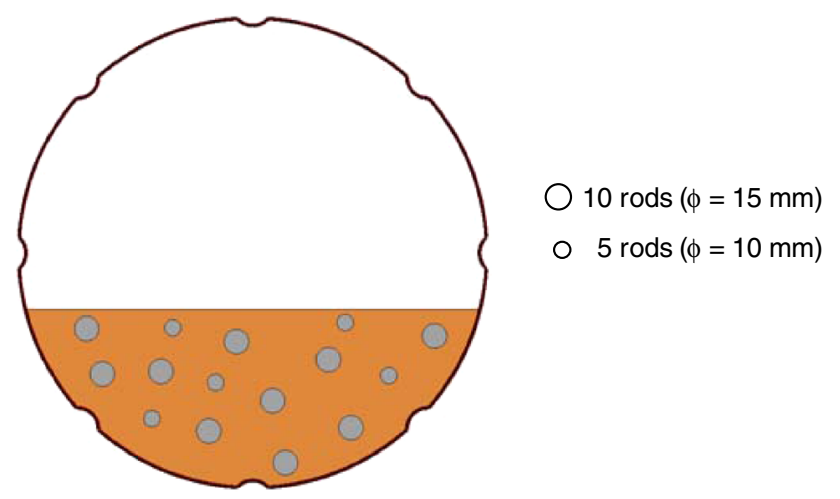

Fig. 18 Numerical model of dry sand and steel rods on a tumbling ball mill

overall results in terms of power consumption can be deemed reasonably accurate.

\subsubsection{Inclusion of milling balls}

Comminution of granular material within a rotating drum by crushing caused by the impact of steel balls is a problem

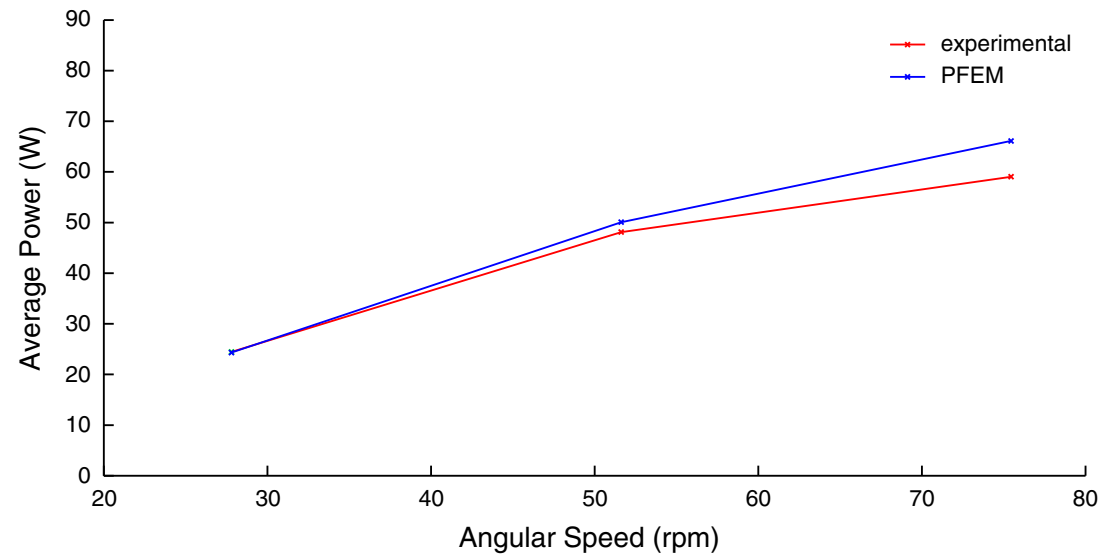

of high interest for many industries (ceramics, composites, foods, minerals, paints, inks and pharmaceuticals and so on). As a first step towards the full simulation of this, admittedly challenging problem, we explore in this example the possibilities of the proposed numerical model to capture, at least, the mechanical interaction between several (hard) bodies and dry sand (crushing effects are, thus, not contemplated in the simulation). The charge for the model is formed by the same amount of dry sand than in previous examples and fifteen rods of two different diameters, namely, 10 rods of $\phi_{1}=15 \mathrm{~mm}$ and 5 rods of $\phi_{2}=10 \mathrm{~mm}$, see Fig. 18. The material and numerical parameters are the same of those given in the introduction of Sect. 3.4.

Since there is no available experimental data for this simulation, the mechanical response of the charge, including the steel rods, is compared with the numerical simulation defined previously as the reference example, Fig. 16(a) (with only dry sand). Figure 19(a) shows a snapshot of the pressure field in the stationary regime. The rods define local areas where the pressures present the maximum values-it is found on the material located between the rods and the drum wall and between the rods themselves.
Fig. 19 Mechanical variables in the charge during motion: a volumetric pressures in the material, b plastic factor
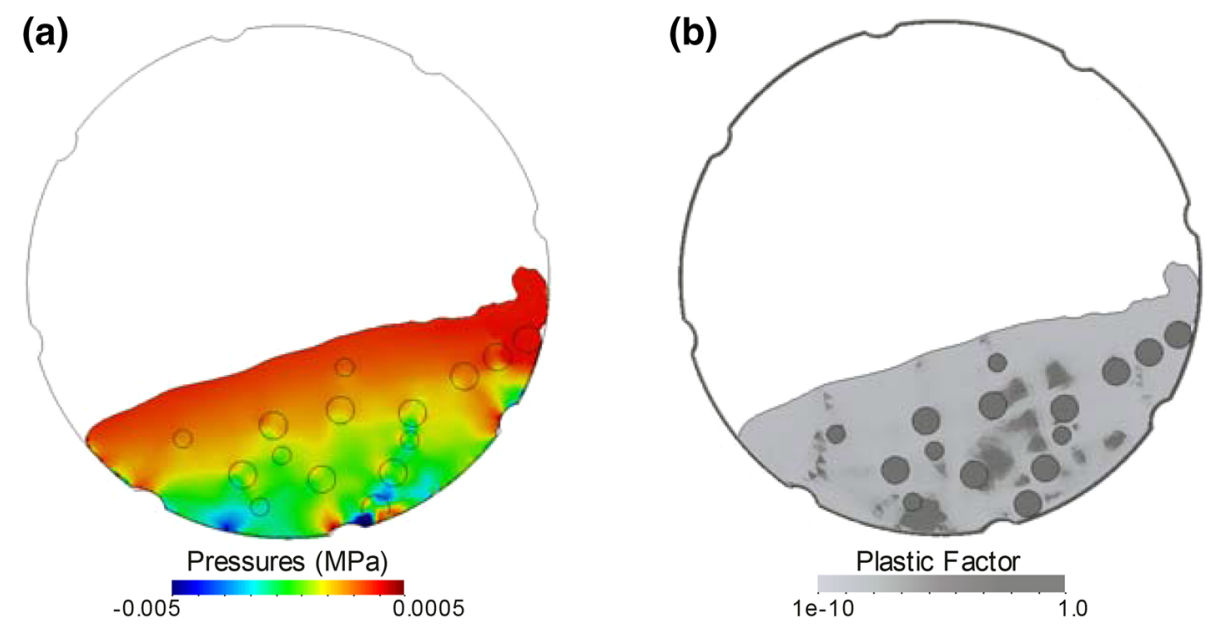
Fig. 20 Torque comparison for a charge at $35 \%$ of degree of filling at $35 \%$ of the critical speed, with and without steel rods

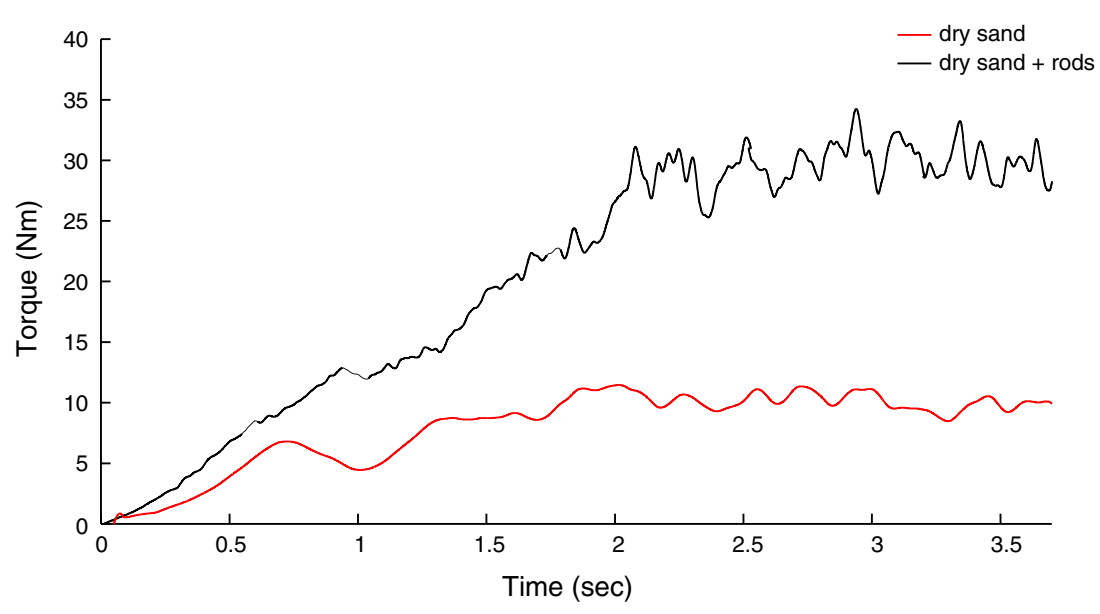

The influence of the rods on the material is observed via the plastic multiplier. Since the model is formulated in the framework of plasticity, this parameter identifies the material in the plastic or elastic regime. For the charge conformed by dry sand, the plastic multiplier lies on the range of full plasticity while for the material interacting with the rods, zones where the material does not reach completely plasticity can be appreciated. Figure 19(b), shows this concept by means of a factor, defined herein as $\alpha=(1+\Delta \lambda)^{-1}$, where $\Delta \lambda$ denotes the plastic multiplier; $\alpha=0$ denotes full plasticity and $\alpha=1$ denotes full elastic regime.

Figure 20 shows the numerical comparison of both charges (with and without steel rods) for a degree of filling of the drum of $35 \%$ and using the referential rotational speed of $35 \%$ of the critical speed. It may be observed that, as it could be expected, the torque of the charge with the steel rods increases significantly. Likewise, the torque in the case of dry sand with steel rods shows a higher degree of oscillations during the motion of the charge, a fact that may be attributable to the displacement and impact of the rods during the process.

\section{Concluding remarks}

Along these work, a PFEM-based model is applied to simulation of two industrial granular flows: silo discharge and tumbling mills. From these simulations, the following conclusions can be obtained:

\section{Silo discharge:}

- The simulations, done on different material parameters and geometry (given by the opening of the outlet), allow understanding the flow mechanisms during the silo discharge. The internal friction coefficient affects directly the definition of a mass or a funnel flow; for small values, the mass flow is predominant, while, for larger values, the funnel flow is recovered.

- Despite the type of flow is a function of the internal friction, the opening of the outlet plays a fundamental role.
The spreading of the core of the funnel flow is smaller as long as the opening of the outlet remains small; for larger openings, the funnel tends to open toward the walls at the upper levels of the material.

- Though the wall pressures could not be compared with experimental results, since they were not reported for this problem, it is possible to state a good agreement of the bulk pressure provided by the model and the theoretical hydrostatic pressures on a fluid. Once the flow patterns are validated, it is possible to determine the bulk pressures and the stresses exerted by the material on the walls.

\section{Tumbling mill:}

- Tumbling mill processes involve, in general, rapid granular flows, which are not, in principle, full candidates to be modeled by the considered model. In spite of this, the numerical model presented herein shows a well agreement between experimental and numerical simulations.

- The comparison of the consumption of the power of the system shows a higher degree of accuracy for lower rotational speeds, which indicates that the simulations for large rotational speeds could be improved by enriching the constitutive model allowing to simulate rapid granular flows.

The proposed constitutive model shows a good ability to predict the kinematic behavior of dense granular flows and to define stagnant and flow zones, as well as the transition of jamming in the material. Simulations of both types of industrial processes state the potential of the proposed constitutive model, in combination with the Particle Finite Element Method, resulting in a robust numerical tool, suitable to handle dense granular flows in industrial processes.

Acknowledgments Authors, J. Oliver, J.C. Cante, and J. A. Hernández, acknowledge the financial support from the European Research Council, under the (FP/2007-2013)/ERC Grant Agreement no. 320815, 
Advanced Grant Project COMP-DES-MAT, and the Spanish Ministry of Science and Innovation under grant BIA2011-24258. The authors also acknowledge the partial financial support from Hjalmar Lundbohm Research Center and LKAB.

\section{References}

1. Ravenet J (1981) Silos problems. Bulk Solids Handl 1(4):667-679

2. Nedderman RM (2005) Statics and kinematics of granular materials. Cambridge University Press, New York

3. Zhang X, Krabbenhoft K, Pedroso D, Lyamin A, Sheng D, Da Silva MV, Wang D (2013) Particle finite element analysis of large deformation and granular flow problems. Comput Geotech 54: 133-142

4. Chen JF, Rotter JM, Ooi JY, Zhong Z (2007) Correlation between the flow pattern and wall pressures in a full scale experimental silo. Eng Struct 29:2308-2320

5. Clermont B, de Haas B (2010) Optimization of mill performance by using online ball and pulp measurements. J South Afr Inst Min Metall 110:8

6. Tano K (2005) Continuous monitoring of mineral processes with special focus on tumbling mills: a multivariate approach. Doctoral thesis, Luleå University of Technology

7. Si G, Cao H, Zhang Y, Jia L (2009) Experimental investigation of load behaviour of an industrial scale tumbling mill using noise and vibration signature techniques. Miner Eng 22:1289-1298

8. Jonsén P, Pålsson BI, Tano K, Berggren A (2011) Prediction of mill structure behaviour in a tumbling mill. Miner Eng 24:236-244

9. Jonsen P, Stener J, Palsson B, Haggblad H (2013) Validation of tumbling mill charge-induced torque as predicted by simulations. Miner Metall Process J 30:220-225

10. Duran J (2009) Sands, powders, and grains: an introduction to the physics of granular materials (partially ordered systems). Springer, New York

11. Belytschko T, Liu WK, Moran B (2000) Nonlinear finite elements for continua and structures. Wiley, New York

12. Idelsohn SR, Oñate E, Pin FD (2004) The particle finite element method: a powerful tool to solve incompressible flows with freesurfaces and breaking waves. Int J Numer Method Eng 61:964-989

13. Edelsbrunner H, Mücke EP (1994) Three-dimensional alpha shapes. ACM Trans Graph 13:43-72

14. Oñate E, Rossi R, Idelsohn SR, Butler KM (2010) Melting and spread of polymers in fire with the particle finite element method. Int J Numer Method Eng 81:1046-1072

15. Oñate E, Idelsohn S, Celigueta M, Rossi R (2008) Advances in the particle finite element method for the analysis of fluid-multibody interaction and bed erosion in free surface flows. Comput Method Appl Mech Eng 197:1777-1800

16. Carbonell J, Oñate E, Suárez B (2009) Modeling of ground excavation with the particle finite-element method. J Eng Mech 136: 455-463

17. Cante JC, Riera MD, Oliver J, Prado JM, Isturiz A, Gonzalez C (2011) Flow regime analyses during the filling stage in powder metallurgy processes: experimental study and numerical modelling. Granul Matter 13(1):79-92
18. Oliver J, Cante JC, Weyler R, González C, Hernandez J (2007) Particle finite element methods in solid mechanics problems. In: Oñate E, Owen R (eds) Computational plasticity, vol 7. Springer, Netherlands, pp 87-103

19. Larese A, Rossi R, Oñate R, Idelsohn SR (2012) A coupled PFEMEulerian approach for the solution of porous FSI problems. Comput Mech 50:805-819

20. Harr M (2002) Stress distribution, in the Civil Engineering handbook, 2nd edn. CRC Press, Boca Raton

21. Dohrmann CR, Bochev PB (2004) A stabilized finite element method for the Stokes problem based on polynomial pressure projections. Int J Numer Method Fluids 46:183-201

22. Simo JC, Hughes TJR (1998) Computational inelasticity. Springer, New York

23. Huang S (1995) Continuum theory of plasticit. Wiley, New York

24. Hernández J, Oliver J, Cante J, Weyler R (2011) Numerical modeling of crack formation in powder forming processes. Int J Solid Struct 48:292-316

25. Hernández J, Oliver J, Cante J, Weyler R (2012) Finite element modelling of ejection cracks in powder metallurgy die compaction processes: case study. Powder Metall 55:36-44

26. Gustafsson G (2012) Mechanical characterization and modelling of iron ore pellets. Doctoral thesis, Division of Mechanics of Solid Materials, Luleå University of Technology, Sweden

27. Gustafsson G, Häggblad HÅ, Oldenburg M (2007) Smoothed particle hydrodynamic simulation of iron ore pellets flow. In: AIP conference proceedings, $\mathrm{p} 1483$

28. Rycroft C, Kamrin K, Bazant M (2009) Assessing continuum postulates in simulations of granular flow. J Mech Phys Solid 57: $828-839$

29. Oliver J, Huespe AE, Blanco S, Linero DL (2006) Stability and robustness issues in numerical modeling of material failure with the strong discontinuity approach. Comput Method Appl Mech Eng 195:7093-7114

30. Oliver J, Huespe AE, Cante JC (2008) An implicit/explicit integration scheme to increase computability of non-linear material and contact/friction problems. Comput Method Appl Mech Eng 197:1865-1889

31. Oliver J, Hartmann S, Cante JC, Weyler R, Hernández JA (2009) A contact domain method for large deformation frictional contact problems. Part 1: theoretical basis. Comput Method Appl Mech Eng 198:2591-2606

32. Hartmann S, Oliver J, Weyler R, Cante JC, Hernández JA (2009) A contact domain method for large deformation frictional contact problems. Part 2: numerical aspects. Comput Method Appl Mech Eng 198:2607-2631

33. Rotter J, Ooi J, Chen J, Tiley P, Mackintosh I, Bennett F (1995) Flow pattern measurement in full scale silos. The University of Edinburgh, Edinburgh, Scotland

34. Knupp PM (2003) Algebraic mesh quality metrics for unstructured initial meshes. Finite Elements Anal Design 39:217-241

35. Cleary PW (2001) Charge behaviour and power consumption in ball mills: sensitivity to mill operating conditions, liner geometry and charge composition. Int J Miner Process 63:79-114

36. Holzapfel GA (2000) Nonlinear solid mechanics: a continuum approach for engineering. Wiley, New York 